\title{
Role of Solid-Solid Interfacial Energy Anisotropy in the Formation of Broken Lamellar Structures in Eutectic Systems
}

\author{
SUMEET KHANNA, SHANMUKHA KIRAN ARAMANDA, \\ and ABHIK CHOUDHURY
}

\begin{abstract}
Eutectic solidification gives rise to a wide range of microstructures. A commonly observed morphology is the periodic arrangement of lamellar plates with well-defined orientations of the solid-solid interface in a given eutectic grain. It is typically believed that this form of morphology develops due to the presence of solid-solid interfacial energy anisotropy. In this paper, we provide evidence using phase-field simulations where our focus is on alloys where the minority phase fraction is low. Our aim is to establish the role of solid-solid interfacial energy anisotropy in the stabilization of broken lamellar structures in such systems in contrast to the formation of a rod microstructure. In this regard, we conduct phase-field simulations for different strengths of anisotropy in both constrained and extended settings, using which we clarify the mechanisms by which a lamellar arrangement gets stabilized in the presence of anisotropy in the solid-solid interfacial energy.
\end{abstract}

https://doi.org/10.1007/s11661-020-05995-8

(c) The Minerals, Metals \& Materials Society and ASM International 2020

\section{INTRODUCTION}

EUTECTIC alloy solidification as an example of complex pattern formation has been studied by both experimentalists and theoreticians. ${ }^{[1-14]}$ The phase transformation yields a wide range of microstructures like rods, lamellae, labyrinthine, and mixed growth structures during two-phase growth, and the patterns become increasingly complex with the addition of more phases and components. ${ }^{[15-20]}$ While the influence of volume fractions on the morphology is quite well established wherein alloys with minority phase volume fractions lower than the critical value for lamellar to rod transition $(\approx 30 \mathrm{pct}),{ }^{[21]}$ a rod microstructure is observed and close to equal volume fractions yield labyrinthine/lamellar microstructures, yet there exist alloys which show behavior contrary to this rule. Eutectics like $\mathrm{Ag}-\mathrm{Bi},{ }^{[22-27]}$

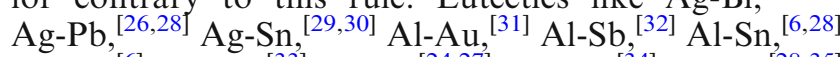
$\mathrm{Bi}-\mathrm{Mg},{ }^{[6]} \mathrm{Bi}-\mathrm{Mn},{ }^{[33]} \mathrm{Bi}-\mathrm{Zn},{ }^{[24,27]} \mathrm{Cd}-\mathrm{Ge},{ }^{[34]} \mathrm{In}-\mathrm{Zn},{ }^{[28,35]}$ $\mathrm{Sn}-\mathrm{Zn}^{\{3,36-42]}$ and some organic systems ${ }^{[43]}$ exhibit a lamellar type of microstructure even with a less than 10 pct volume fraction of the minority phase. It has been proposed by several authors as early as Jackson-Hunt ${ }^{[21]}$

SUMEET KHANNA, ARAMANDA SHANMUKHA KIRAN and ABHIK CHOUDHURY are with the Department of Materials Engineering, Indian Institute of Science, Bangalore, 560012, India. Contact e-mail: abhiknc@iisc.ac.in

Manuscript submitted June 7, 2020. along with experimental evidence provided by Caroli et al. ${ }^{[44]}$ Chadwick, ${ }^{[3]}$ Jaffrey and Chadwick, ${ }^{[37]}$ that the reason for the formation of such lamellar structures could be the presence of strong solid-solid interfacial energy anisotropy where the Wulff plot of the solid-solid interface consists of cusps. The lamellar interface orientations are therefore well defined in a given eutectic grain as those corresponding to the low energy directions in the Wulff plot. The presence of such anisotropy can lead to tilted solidification fronts during thin-film growth and has been studied extensively, ${ }^{[44-49]}$ using experiments and simulations. In bulk solidification, evidence from phase-field simulations also suggest that the presence of anisotropy leads to the formation of lamellar structures instead of labyrinthine morphologies ${ }^{[50]}$ in equal volume fraction alloys. Similarly, utilizing phase-field simulations, Gránásy and colleagues ${ }^{[51]}$ have investigated the effect of anisotropy on the evolution of different eutectic patterns.

Therefore, while there is enough evidence to suggest the influence of solid-solid interfacial energy anisotropy in biasing the morphologies towards a periodic arrangement of lamellae, yet, there seems to be lack of understanding on the mechanisms by which a lamellar microstructure gets stabilized, particularly in alloys where the minority fraction is below the critical value for rod-lamellar transition $(\approx 30 \mathrm{pct})$. This is the main objective of the paper, where using phase-field simulations we investigate the differences in morphological evolution that arise as a result of the presence of 
anisotropy in the solid-solid interfacial energy that leads to the stabilization of the lamellar morphology instead of the rod microstructure that is typically observed with isotropic solid-solid interfaces. The phase-field method provides an ideal methodology for conducting dynamical simulations of phase transformations allowing for tracking of morphological changes during evolution, thereby providing clarity to the underlying mechanisms. For this purpose, we choose to mimic the $\mathrm{Sn}-\mathrm{Zn}$ system, which has a minority phase percentage of around 9 pct and is experimentally known to give structures that are "broken lamellar" instead of rods. In separate experimental work, we have characterized this alloy extensively where we have confirmed the following aspects. The microstructure reveals a general alignment of lamellar plates with a gradual increase in the length of the broken fragments as the solidification progresses, where lengthening occurs by merging of lamellae fragments along well-defined directions for a given eutectic grain. In this alloy, there exists a set of crystallographic orientation relationships between the $\mathrm{Sn}$ and the $\mathrm{Zn}$ phases during eutectic growth. Further, the $\mathrm{Zn}$ phase determines the orientation of the lamellar interface, wherein solidification occurs such that the solid-solid interface is always parallel to the basal planes of the $\mathrm{Zn}$ crystal. As a corollary, therefore, the growth directions remain invariant upon change of heat and solutal flux directions. Since, we did not find strong solid-liquid interfacial anisotropy all of the previous experimental observations point towards the presence of solid-solid interfacial energy anisotropy in this alloy that is also supported by earlier findings from Jaffrey and Chadwick, ${ }^{[37]}$ thus making this a good candidate for the present study.

Following is a brief structure of the paper. We begin with the description of the phase-field model and the simulation setup in Section II, followed by the results elaborating on the range of microstructures that are derived in the phase-field simulations in Section III. We finally conclude in Section IV by combining the numerical results in this paper with observations from separate experimental work (presented elaborately elsewhere) where we summarize the mechanism through which the broken lamellar morphology forms.

\section{NUMERICAL SIMULATIONS}

\section{A. Model Description}

We use a phase-field model based upon the grand potential functional. ${ }^{[52,53]}$ As per this model, the Sn-Zn binary eutectic system is completely described by the following three independent variables, the order parameter $\left(\phi=\left\{\phi_{\alpha}, \phi_{\beta}, \phi_{\gamma}, \ldots, \phi_{N}\right\}\right)$ that is composed of the volume fractions of the $N$ different phases, the diffusion potential $(\mu)$, and the temperature $(T)$. In the following sections, we describe the time evolution equations for these three variables. The three phases $(N=3)$ in the eutectic reaction, namely the $\mathrm{Sn}-\mathrm{Zn}$ liquid, the $\mathrm{Sn}$-solid and the $\mathrm{Zn}$ solid phase are assigned three different phase-fields or order parameters $\left(\phi_{\alpha}\right)$ such that,

$$
\phi_{\alpha} \in[0,1] \text { and } \sum_{\alpha}^{\mathrm{N}} \phi_{\alpha}=1 .
$$

The grand potential functional reads,

$$
\Omega(T, \mu, \boldsymbol{\phi})=\int_{\Omega}\left[\psi(T, \mu, \boldsymbol{\phi})+\epsilon a(\boldsymbol{\phi}, \nabla \boldsymbol{\phi})+\frac{w(\boldsymbol{\phi})}{\epsilon}\right] \mathrm{d} \Omega .
$$

Here, $\epsilon$ is the width of the diffuse interface, which is chosen such that the smallest feature in the resulting morphology is accurately resolved. $w(\boldsymbol{\phi})$ is the double obstacle potential, given as,

$$
w(\phi)= \begin{cases}\sum_{\alpha, N}^{N, N} \quad \frac{16}{\pi^{2}} \gamma_{\alpha \beta} \phi_{\alpha} \phi_{\beta}+\gamma_{\alpha \beta \delta} \phi_{\alpha} \phi_{\beta} \phi_{\delta} & \text { if } \phi \in[0,1], \\ \delta \neq \alpha \neq \beta & \text { otherwise. }\end{cases}
$$

$\gamma_{\alpha \beta}$ is the isotropic surface energy of the $\alpha-\beta$ interface. In the present study, the solid-liquid interfaces are isotropic, while the solid-solid interfaces are anisotropic. $\gamma_{\alpha \beta \delta}$ are third-order terms added in order to suppress the appearance of a third phase at a binary interface. $a(\boldsymbol{\phi}, \nabla \boldsymbol{\phi})$ is the gradient energy term and $\psi(T, \mu, \boldsymbol{\phi})$ is the grand potential which will be described later. The anisotropy in the interface energy is incorporated in the gradient energy term as,

$$
a(\phi, \nabla \phi)=\sum_{\alpha<\beta}^{N, N} \gamma_{\alpha \beta}\left[a_{\mathrm{c}}\left(\mathbf{q}_{\alpha \beta}\right)\right]^{2}\left|\mathbf{q}_{\alpha \beta}\right|^{2},
$$

where $a_{\mathrm{c}}$ is the anisotropy function of the $\alpha-\beta$ interface normal vector $\mathbf{q}_{\alpha \beta}=\phi_{\alpha} \nabla \phi_{\beta}-\phi_{\beta} \nabla \phi_{\alpha}$. We utilize an interfacial energy anisotropy function that has a 2-fold symmetry for orientations of the solid-solid interface normal that are perpendicular to the growth direction, where we use the following expression, as described in Reference 50:

$$
a_{\mathrm{c}}=1+\delta_{\alpha \beta}\left(\frac{q_{y}^{\prime 2}-q_{x}^{\prime 2}}{q_{x}^{\prime 2}+q_{y}^{\prime 2}+q_{z}^{\prime 2}}\right)
$$

where $\delta_{\alpha \beta}$ is the strength of anisotropy. $q_{x}^{\prime}, q_{y}^{\prime}, q_{z}^{\prime}$ are the components of $\mathbf{q}_{\alpha \beta}^{\prime}$ along the $x, y$ and $z$ directions. $\mathbf{q}_{\alpha \beta}^{\prime}$ is obtained by rotating $\mathbf{q}_{\alpha \beta}$ by an angle $\theta_{\mathrm{R}}$ about the growth direction ( $Z$ axis), where $\theta_{\mathrm{R}}$ is the orientation angle of the interface anisotropy function. The solidsolid interfacial energy polar plots for two different orientations of the rotation angle $\theta_{\mathrm{R}}=0 \mathrm{deg}$ and $90 \mathrm{deg}$ are shown in Figure 1 along with their corresponding equilibrium shapes (Wulff construction). For stable minimization of the grand potential functional, $\delta_{\alpha \beta}$ in our phase-field model can have a maximum value of $1 / 3$ for the 2-fold anisotropy function. ${ }^{[54,55]}$ We note that the choice of the 2-fold anisotropy is the minimal symmetry that is observed experimentally in this alloy system, for orientations of the lamellar interface normal, perpendicular to the growth direction. However, the complete Wulff plot in 3D of the solid-solid interface is unavailable to us either experimentally or through other lower length-scale modeling routes. In light of this, we have 
(a)

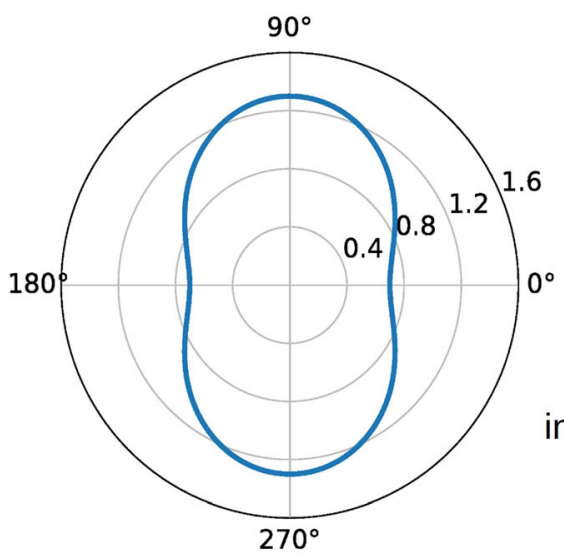

(c)

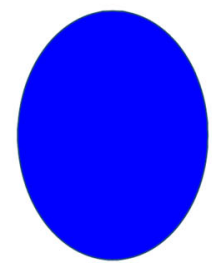

(b)

$$
\theta_{R}=90^{\circ}
$$

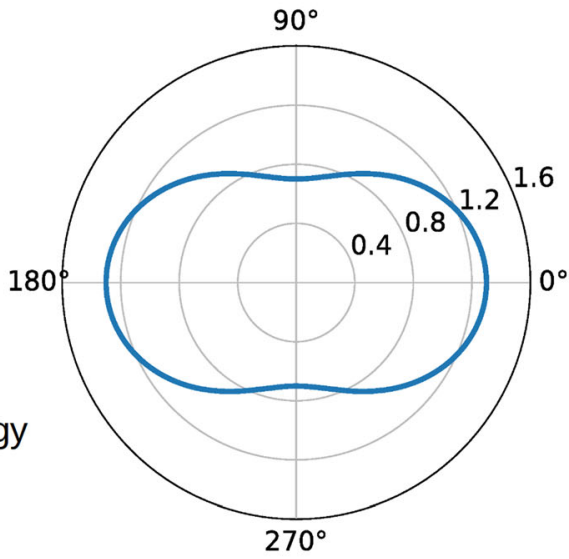

(d)

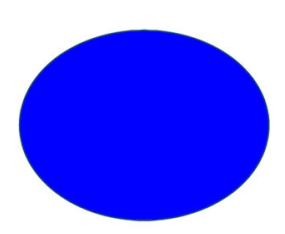

Fig. 1-Polar plots $(a)$ and $(b)$ of the 2-fold solid-solid interfacial energy anisotropy function, plotted for solid-solid interface normals/directions that lie in the transverse plane perpendicular to the solidification direction. Two orientations of the solid-solid interfacial anisotropy function, corresponding to rotations about the growth direction, are shown, where (a) $\theta_{\mathrm{R}}=0$ deg and (b) $\theta_{\mathrm{R}}=90$ deg and strength $\delta_{\alpha \beta}=0.3$. The filled ellipses in $(c)$ and $(d)$ are the transverse cross-sections of the equilibrium Wulff shapes (schematic) corresponding to the two rotation angles (orientations) of the anisotropy function.

presented the following results using a simplified anisotropic form.

$\psi$ is the grand potential density, which is obtained at any point as a weighted sum of the grand potential densities of each phase present at that point,

$$
\psi=\sum_{\alpha}^{N} h_{\alpha}(\boldsymbol{\phi}) \psi^{\alpha},
$$

where $h_{\alpha}(\phi)$ is a third-order interpolation function that reads,

$$
h_{\alpha}(\boldsymbol{\phi})=3 \phi_{\alpha}^{2}-2 \phi_{\alpha}{ }^{3}+2 \phi_{\alpha} \sum_{\substack{\beta, \gamma \neq \alpha \\ \beta<\gamma}}^{N, N} \phi_{\beta} \phi_{\gamma} .
$$

$\psi^{\alpha}=\psi^{\alpha}(\mu, T)$ is the grand potential density of phase $\alpha$ and can be written as

$$
\psi^{\alpha}(T, \mu)=f^{\alpha}\left(c^{\alpha}(T, \mu)\right)-\mu c^{\alpha}(T, \mu),
$$

where $f^{\alpha}$ is the Helmholtz free energy density per unit volume. Under constant pressure and volume, $f^{\alpha}$, the Helmholtz free energy density differs from the Gibbs free energy density by a constant. ${ }^{[56]}$ We use a parabolic approximation to the free energies obtained from the COST-507 database ${ }^{[57]}$ and incorporate in the phasefield model similarly as References 49,58 . The BCT A5 phase and the HCP ZN phase from the database are called the $\mathrm{Sn}$ and $\mathrm{Zn}$ phases in the paper, respectively. The parabola for each of the phases is of the form,

$$
f^{\alpha}=A^{\alpha}\left(c^{\alpha}\right)^{2}+B^{\alpha}(T) c^{\alpha}+D^{\alpha}(T),
$$

where the coefficients $A^{\alpha}, B^{\alpha}(T)$ and $D^{\alpha}(T)$ are derived such that the reproduced phase diagrams have the correct equilibrium composition, temperature, liquidus slopes, while the Gibbs-Thomson coefficient is accurately reproduced at leading order at the eutectic point. The liquidus slopes for any $\alpha-l$ phase equilibrium will be denoted as $m^{l-\alpha}$, while the corresponding solidus slope as $m^{\alpha-l}$. The value of $A^{1}$ is derived directly from the second derivative of the free energy vs composition diagram of the liquid, computed at the eutectic composition as,

$$
A^{1}=\frac{\partial^{2} f^{1}}{\partial c^{1^{2}}}
$$

and this same value is used for all the solid phases. This is done to increase the efficiency of the phase-field simulations in terms of optimizing the possible time-step for stable temporal integration. We also note that since at leading order the Gibbs-Thomson coefficient of each of the solid phases may be written as, $\Gamma^{\alpha}=\frac{\gamma_{\alpha l} m^{l-\alpha}}{\frac{\partial^{2} f^{1}}{\partial c^{2}}\left(c_{\text {eut }}^{\alpha}-c_{\text {eut }}^{1}\right)}$, where $c_{\text {eut }}^{\alpha}, c_{\text {eut }}^{1}$ are, respectively, 
the $\alpha$ and liquid phase compositions at the eutectic temperature; we expect to retrieve the correct Gibbs-Thomson effect with respect to the shift of the liquid compositions in equilibrium with the solid, since the interfacial properties such as the solid-liquid interfacial energies as well as the thermodynamic parameters such as the liquidus slopes, equilibrium compositions, and the second derivative of the liquid free energies are derived from either databases or from literature. Taking $B^{\mathrm{l}}(T)=0$ and $D^{\mathrm{l}}(T)=0$, we obtain the coefficients of the parabola corresponding to the solid phases as,

$$
B^{\alpha}(T)=2 A^{1} c_{\text {eut }}^{1}-2 A^{\alpha} c_{\text {eut }}^{\alpha}+\left(T-T_{\text {eut }}\right)\left[\frac{2 A^{1}}{m^{l-\alpha}}-\frac{2 A^{\alpha}}{m^{\alpha-l}}\right],
$$

and

$$
\begin{aligned}
D^{\alpha}(T)= & -A^{1} c_{\text {eut }}^{1}{ }^{2}+A^{\alpha} c_{\text {eut }}^{\alpha{ }^{2}}+(T \\
& \left.-T_{\text {eut }}\right)\left[-\frac{2 A^{1} c_{\text {eut }}^{1}}{m^{l-\alpha}}+\frac{2 A^{\alpha} c_{\text {eut }}^{\alpha}}{m^{\alpha-l}}\right],
\end{aligned}
$$

where $T_{\text {eut }}$ is the eutectic temperature. The reproduced phase diagram constructed using the parabolic free energies is shown in Figure 2(b). The equilibrium volume fraction of the phases in this phase diagram differs from the actual value because of the assumption of equal densities of all phases. Hence, the volume fraction of the phases used in the phase-field model is 14 pct, while it is around 9 pct in reality. However, since both these volume fractions fall well below the limit $(\approx 30 \mathrm{pct})^{[21]}$ where the rod morphology is expected to be stable instead of lamellae for isotropic interfacial energies, we will assume its influence on the eventual microstructural evolution to be insignificant for the scope of this study. The values of the parameters used in the phase-field model are listed in Table I.

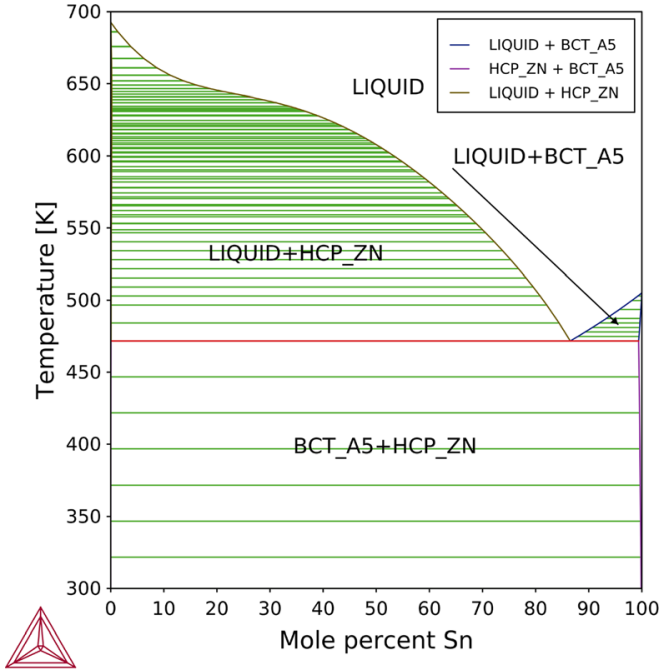

(a)
The minimization of the grand potential functional in Eq. [1] drives the system towards equilibrium. The simplest equation which definitely decreases the grand potential functional with time is given by the AllenCahn type equation that for multiple phases is written as,

$$
\begin{aligned}
\tau \epsilon \frac{\partial \phi_{\alpha}}{\partial t}= & \epsilon\left(\nabla \cdot \frac{\partial a(\phi, \nabla \phi)}{\partial \nabla \phi_{\alpha}}-\frac{\partial a(\phi, \nabla \phi)}{\partial \phi_{\alpha}}\right) \\
& -\frac{1}{\epsilon} \frac{\partial w(\phi)}{\partial \phi_{\alpha}}-\frac{\partial \Psi(T, \mu, \phi)}{\partial \phi_{\alpha}}-\lambda,
\end{aligned}
$$

where $\tau$ is the relaxation constant which controls the kinetics of the phase evolution equation and is interpolated at the interface as $\frac{\sum_{\alpha, \beta}^{N, N} \tau_{\alpha \beta} \phi_{\alpha} \phi_{\beta}}{\sum_{\alpha, \beta}^{N, N} \phi_{\alpha} \phi_{\beta}}$, where $\tau_{\alpha \beta}$ corresponds to the relaxation constant of the $\alpha-\beta$ interface. The values of $\tau_{\alpha \beta}$ are chosen according to the thin-interface asymptotic analysis performed in Reference 52, such that diffusion-controlled growth may be achieved for the solid-liquid interfaces, while the lowest value among the two solid-liquid interfaces is chosen for the solid-solid interface, such that the relaxation of the solid-solid interfaces is always faster than the solid-liquid interfaces. Also, at points where $\sum_{\alpha, \beta}^{N, N} \phi_{\alpha} \phi_{\beta}=0, \tau=\min \left(\tau_{\alpha l}\right) . \lambda$ is the Lagrange multiplier utilized for imposing the constraint $\sum_{\alpha}^{N} \phi_{\alpha}=1$, i.e., the sum of volume fraction of all the phases is 1 . This equation is solved in a coupled manner along with the mass conservation equation. The evolution equation for the diffusion potential $\mu$ of the independent component $(\mathrm{Sn})$ that ensures mass conservation is written as,

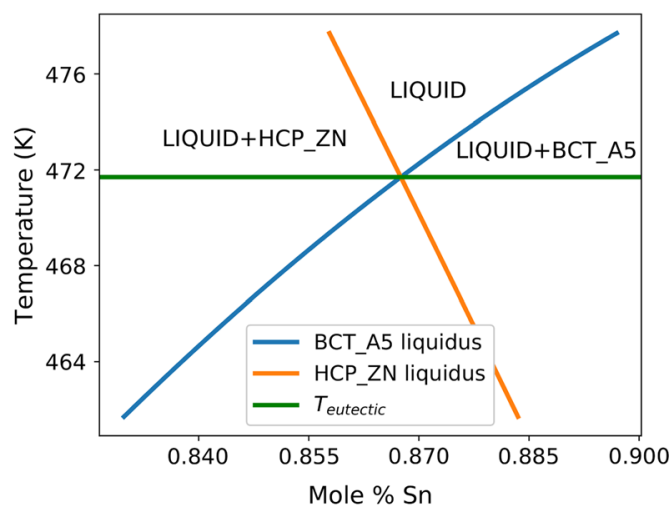

(b)

Fig. 2-Sn-Zn phase diagram, BCT_A5 is the Sn phase, and HCP_Zn is the $\mathrm{Zn}$ phase. (a) Phase diagram generated using the Thermo-Calc software ${ }^{[59]}$ (b) Reproduced phase diagram from parabolic free energies showing liquidus below the eutectic temperature, zoomed in plot around the eutectic point. 
Table I. Simulation Parameters Calculated Using the Physical Values from Refs. [40] and [60], the Procedure for Non-dimensionalization is Described in Appendix A.

\begin{tabular}{|c|c|c|c|}
\hline Parameter & Symbol & Value (Non-dimensional) & Value (Dimensional) \\
\hline Eutectic Temperature & $T_{\text {eut }}$ & 1 & $471.7 \mathrm{~K}$ \\
\hline \multirow[t]{3}{*}{ Eutectic Composition (Mol. Fr. Sn) } & $c_{\mathrm{eut}}^{\mathrm{l}}$ & 0.8675 & 0.8675 \\
\hline & $c_{\text {eut }}^{\mathrm{Sn}}$ & 0.9942 & 0.9942 \\
\hline & $c_{\mathrm{eut}}^{\mathrm{Zn}}$ & 0.0007 & 0.0007 \\
\hline \multirow[t]{2}{*}{ Liquidus Slope } & $m^{1-S n}$ & 0.5 & $235.85 \mathrm{~K} \mathrm{~mol}^{-1}$ \\
\hline & $m^{1-\mathrm{Zn}}$ & -1.30 & $-613.21 \mathrm{~K} \mathrm{~mol}^{-1}$ \\
\hline \multirow[t]{4}{*}{ Interface Energy } & $\gamma_{\mathrm{Sn}-1}$ & 1.0 & $0.104 \mathrm{~J} \mathrm{~m}^{-2}$ \\
\hline & $\gamma_{\mathrm{Zn}-1}$ & 0.782 & $0.0813 \mathrm{~J} \mathrm{~m}^{-2}$ \\
\hline & $\gamma_{\mathrm{Sn}-\mathrm{Zn}}$ & 1.46 & $0.1519 \mathrm{~J} \mathrm{~m}^{-2}$ \\
\hline & $\gamma_{\alpha \beta \delta}$ & 15 & - \\
\hline Isotropic Interface Width & $W$ & 10.8 & $4.6 \times 10^{-9} \mathrm{~m}$ \\
\hline Diffusivity & $D^{1}$ & 1.0 & $3.5 \times 10^{-9} \mathrm{~m}^{2} \mathrm{~s}^{-1}$ \\
\hline Velocity & $v$ & 0.005 & $4.1 \times 10^{-2} \mathrm{~m} \mathrm{~s}^{-1}$ \\
\hline
\end{tabular}

$$
\frac{\partial \mu}{\partial t}=\frac{\nabla \cdot M \nabla \mu-\nabla \cdot\left(\mathbf{j}_{\mathrm{at}}\right)-\sum_{\alpha} h_{\alpha}(\boldsymbol{\phi}) \frac{\partial c^{\alpha}}{\partial T} \frac{\partial T}{\partial t}-\sum_{\alpha} c^{\alpha}(T, \mu) \frac{\partial h_{\alpha}(\boldsymbol{\phi})}{\partial \phi_{\alpha}} \frac{\partial \phi_{\alpha}}{\partial t}}{\sum_{\alpha} h_{\alpha}(\boldsymbol{\phi}) \frac{\partial c^{\alpha}}{\partial \mu}},
$$

where for parabolic free energies, we have,

$$
\begin{gathered}
\frac{\partial c^{\alpha}}{\partial \mu}=\frac{1}{2 A^{\alpha}}, \\
c^{\alpha}=\frac{\mu-B^{\alpha}(T)}{2 A^{\alpha}} .
\end{gathered}
$$

Additionally, the derivative of the phase composition with temperature is derived as,

$$
\frac{\partial c^{\alpha}}{\partial T}=\frac{1}{m^{l-\alpha}},
$$

where $m^{l-\alpha}$ is the liquidus slope of phase $\alpha . \mathbf{j}_{\text {at }}$ is the antitrapping current that is added to the flux for incorporating asymptotic corrections in a one-sided diffusion problem. $\mathbf{j}_{\text {at }}$ is a flux directed from the solid side of the interface to the liquid side in the direction of the interface normal as,

$$
\mathbf{j}_{\mathrm{at}}=\frac{\pi \epsilon \phi_{\alpha}\left[1-h_{\alpha}(\boldsymbol{\phi})\right]}{4 \sqrt{\phi_{\alpha}\left(1-\phi_{\alpha}\right)}} \epsilon\left[c^{\beta}(T, \mu)-c^{\alpha}(T, \mu)\right] \frac{\partial \phi_{\alpha}}{\partial t} \frac{\nabla \phi_{\alpha}}{\left|\nabla \phi_{\alpha}\right|},
$$

where $\beta$ represents the liquid phase, and $\alpha$ represents the solid phases.

\section{B. Simulation Setup}

The phase-field, composition, and temperature evolution equations described above are solved in a coupled manner using forward Euler method in time discretized on a $3 \mathrm{D}$ staggered grid. The simulation results have been verified for different interface widths and grid resolution for numerical consistency in Appendix B.. The code is parallelized using MPI with parallel file $\mathrm{I} / \mathrm{O}$ using the parallel HDF5 library ${ }^{[61]}$ to run large simulations efficiently across multiple processors. ParaView ${ }^{[62,63]}$ and matplotlib, ${ }^{[64]}$ scikit-image, ${ }^{[65]}$ and PyMKS ${ }^{[66,67]}$ libraries in Python are used for post-processing and visualization of simulation output.

Jackson and Hunt ${ }^{[21]}$ proposed a relationship between the average interface undercooling and the characteristic spacing during coupled growth of two-phase eutectic alloys, which can be utilized for deriving information about the possible morphologies that might be observable in experiments. Additionally, the expression for the undercooling at the interface may be utilized for the calculation of the shape of the solid-liquid interface. In the following section, we perform these calculations using the phase-field method where we compute the interface shapes and the resultant interfacial undercooling for different characteristic spacings in the presence of solid-solid interfacial energy anisotropy. These simulations are going to be performed in constrained 3D domains as in Reference 68. Later, we perform extended simulations comparable to experimental conditions in order to investigate realistic microstructure evolution. Following is a brief description about the simulation settings.

Constrained simulations enable us to specifically study the effect of interface energy anisotropy on the eutectic morphology. These simulations are initialized with a hexagonal configuration of $\mathrm{Zn}$ rods in the $\mathrm{Sn}$ matrix. We choose this initial hexagonal configuration as it is the stable one for isotropic interfaces when the minority phase volume fraction is low. Due to hexagonal symmetry, we choose a small rectangular domain as shown in Figure 3(a) with reflective boundary condition applied across the cross-section. This cross-section is depicted as the white rectangle in Figure 3(b). On mirroring the cross-section about the boundaries, the hexagonal configuration is reproduced as shown in Figure 3(b) (in green). We have also depicted the Cartesian coordinate system in Figure 3(b) 


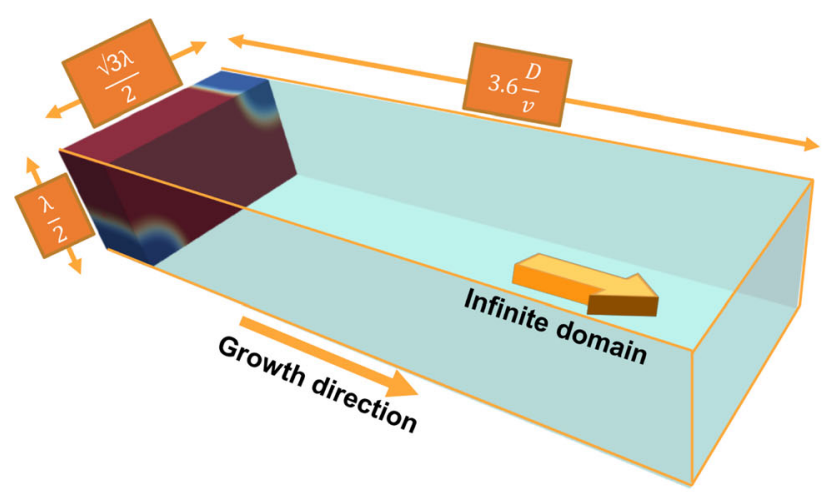

(a)

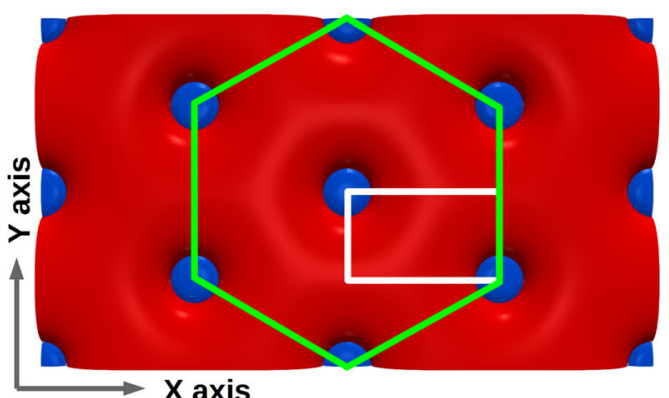

(b)

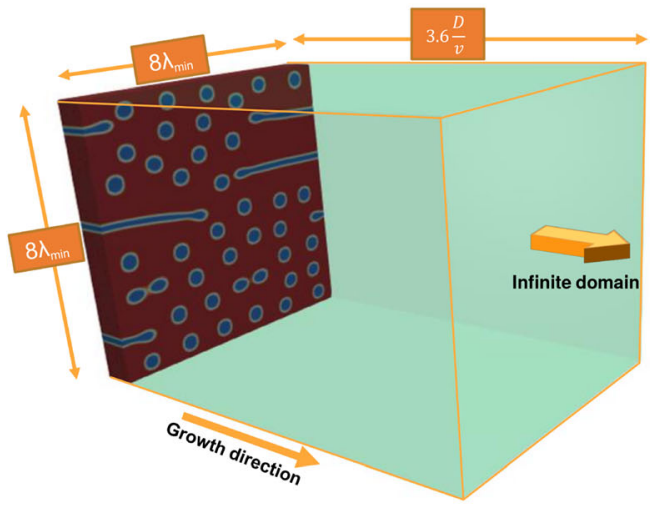

(c)

Fig. 3-Simulation setups that are utilized for the constrained $(a, b)$ and extended $(c)$ simulations, where red $=\mathrm{Sn}$ phase, blue $=\mathrm{Zn}$ phase. (a) $\lambda$ is the initial spacing for the rod morphology. Reflective boundary condition is applied on the cross-section boundaries, where the simulated region in (a) corresponds to the region in the white rectangle in (b). On mirroring about the four edges of the white rectangle, the hexagonal arrangement of $\mathrm{Zn}$ rods is obtained. The $X$ and the $Y$ axes are as depicted in (b), and the $Z$ axis (growth direction) is out of the plane of paper. (c) Simulation setup for extended simulation, reflective boundary condition is applied on the cross-section boundaries (Color figure online).

which we will follow throughout the paper, the growth axis is along the $Z$ direction (out of paper), and the $X$ and $Y$ axes are in the plane of cross-section (transverse plane). The influence of the solid-solid interfacial energy anisotropy function on the morphology is determined by symmetry, strength, as well as the orientation of the anisotropy with respect to the simulation domain. Thereby, we perform simulations by varying the strength of the anisotropy and the orientation angle, $\theta_{\mathrm{R}}=0 \mathrm{deg}$ and $90 \mathrm{deg}$ (see the section on model formulation for details) for the 2-fold anisotropy function. While these simulations would let us identify the different morphologies that are possible, the steady state attained in these constrained simulations might not be a true reflection of the possibilities in an extended simulation due to the restricted degrees of freedom. However, the effect of changing the anisotropy parameters on the morphology is evident from the constrained simulation results. In order to get a more realistic picture akin to the experimental conditions, we perform extended directional growth simulations as described in Figure 3(c). We initialize with a random distribution of $\mathrm{Zn}$ rods in the $\mathrm{Sn}$ matrix. In this case, all the $\mathrm{Zn}$ rods have the same orientation relationship with the $\mathrm{Sn}$ phase. The domain length for both of the above simulation conditions in the growth direction is taken to be 600 grid points, which is $\approx 3.6 \mathrm{D} / \mathrm{v}$, long enough to mitigate boundary effects on the liquid diffusion profile. Since we are simulating solidification from an infinite reservoir of liquid, the far-field liquid composition should remain uninfluenced from the interfacial mass diffusion as the solid grows. Thus, we have implemented regular shifts against the growth direction, ${ }^{[69]}$ in which solidified cells at the start of the simulation box are removed and liquid cells of initial composition are added to the end of the simulation box. In order to impose directional growth at a constant velocity, we impose a moving temperature gradient given as,

$$
T(z, t)=T_{0}+G_{\mathrm{T}}(z-v t) .
$$

The thermal diffusivity value is typically about three orders of magnitude larger than the compositional diffusivity, ${ }^{[70]}$ resulting in a 3 orders faster temperature readjustment compared to the composition profiles. Due to this rapid thermal diffusion w.r.t. to solute diffusion, we can assume that the temperature is always 
at steady state, which in the case of directional solidification is the externally imposed linear temperature gradient and thereby the rate of change of temperature is,

$$
\frac{\partial T}{\partial t}=-G_{\mathrm{T}} v,
$$

which is an input in Eq. [13].

\section{RESULTS AND DISCUSSIONS}

\section{A. Constrained Simulations}

\section{Undercooling vs spacing}

We begin with constrained simulations, firstly to identify the undercooling $v s$ spacing relationships in the presence of anisotropy, secondly to investigate the change in the shape of the solid-liquid interfaces of the rod phase as a function of spacing, and thirdly to ascertain the stability of the hexagonal configuration towards either change in the arrangement of the rods or the formation of lamellae as the spacing is changed. In order to derive the undercooling $v s$ spacing relationship, we initialize the hexagonal rod configuration with different spacings. For smaller spacings, the final steady-state morphology remains as a hexagonal array of rods, with modifications in the shapes of the $\mathrm{Zn}$ rods. The undercooling $v s$ spacing relations for the three cases: isotropic, anisotropic with $\left(\theta_{\mathrm{R}}=0 \mathrm{deg}\right)$, and anisotropic with $\left(\theta_{\mathrm{R}}=90 \mathrm{deg}\right)$ are depicted in Figure 4, where the simulation points are fitted with the expression $\Delta T=k_{1} v \lambda+k_{2} / \lambda, v$ being the velocity and $\lambda$ the rod spacing. We see that the curves for the two anisotropic cases almost overlap and the minimum undercooling spacing $\left(\lambda_{\min }^{\text {aniso }}=62\right)$ is almost the same with the isotropic case $\left(\lambda_{\text {min }}^{\text {iso }}=64\right)$. However, the minimum undercooling for the anisotropic case is slightly lower than the isotropic case. The analytical $\lambda_{\text {min }}$ calculated for the isotropic case following the

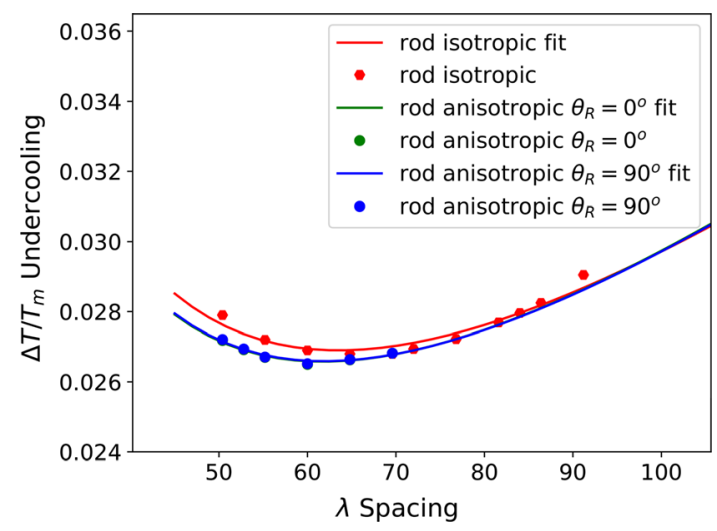

Fig. 4-Undercooling vs spacing curves for the rod morphology for three different cases; isotropic, anisotropic solid-solid interfacial energies with orientation of the anisotropy function $\theta_{\mathrm{R}}=0 \mathrm{deg}$ and $90 \mathrm{deg}$. The points for the two anisotropic cases overlap for most spacings.
Jackson-Hunt derivation is 78, and the difference in the minimum undercooling with respect to the simulated one is $<1$ pct. Throughout the paper, we have normalized $\lambda$ with respect to $\lambda_{\text {min }}^{\text {iso }}=64$, unless explicitly stated otherwise.

\section{Steady-state shapes: small spacings}

Figure 5 represents the steady-state morphology showing the top view of the solid-liquid interface for an initial spacing of $\lambda=69.6$, which corresponds to $1.09 \lambda_{\min }^{\text {iso }}$ or $1.12 \lambda_{\text {min }}^{\text {aniso }}$. The steady-state shapes of the rods within the stability limit (stability towards branching) are characterized by the aspect ratio of the rod cross-section, which is plotted in Figure 5(d). For lower spacings, the aspect ratio tends towards that given by the Wulff shape of an isolated rod under the same anisotropy conditions, where the $\mathrm{Zn}$ rods become elongated such that the interfaces with the lower energy have larger interface areas as depicted in the Wulff shapes in Figures 1(c) and (d). However, with increase in the spacings, the cross-sectional shape of the rods deviates from the equilibrium Wulff shape and actually tends towards circular shapes, where there is also an apparent symmetry with respect to the rod morphologies derived for the two different orientations of the solid-solid interfacial energy anisotropy $\left(\theta_{\mathrm{R}}\right)$.

The individual shapes of the solid-liquid interfaces as well as the triple lines are depicted in Figure 6, where one of the striking differences from the case of isotropic interfacial energy is that the triple-line contour does not lie in a single plane, which implies that the points on the triple line are not corresponding to the same undercooling. This can be understood clearly on the basis of the variation of the solid-solid interfacial energy where the orientations corresponding to higher solid-solid interfacial energies are also the directions where the triple line is depressed more with respect to the average position of the solidification front and thereby also are regions of higher undercooling. The orientations for which the solid-solid interfacial energies are high are also the parts of the solid-liquid interface, we expect the curvature undercooling to be larger and thereby because of this variation of undercooling with orientation, the change in the shape of the triple line with respect to the isotropic case is expected. Moreover, we see that with an increase in spacing, the shape of the rod-liquid interface becomes flatter near the center of the rod with only one of the principal curvature values being non-zero. The situation thus tends towards the case of a lamella with a single non-zero principal curvature as seen in Figure 7 (right column) for larger spacings in comparison to the smaller ones [Figure 7 (left column)].

We note that the changes in the shapes of the rods can also arise in isotropic situations but these occur for spacings much larger than the minimum undercooling spacing. ${ }^{[68]}$ These shape changes result out of a shape bifurcation leading to elongation either towards the first or the second nearest neighbors in the hexagonal rod configuration. Given that the elongations are not symmetric, the shape bifurcation behavior is different for the elongation of the rod morphologies in the two 


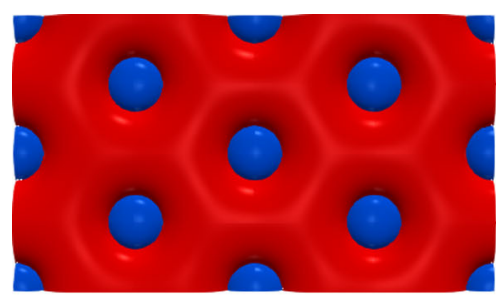

(a)

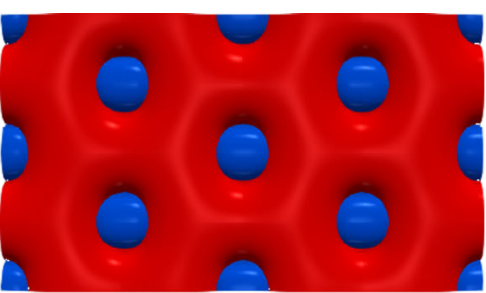

(b)

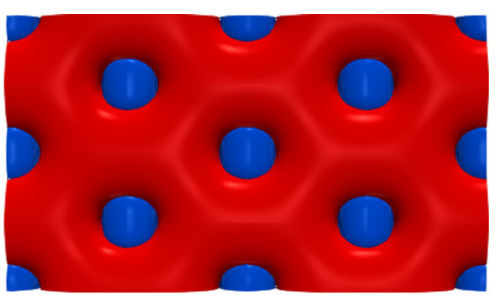

(c)

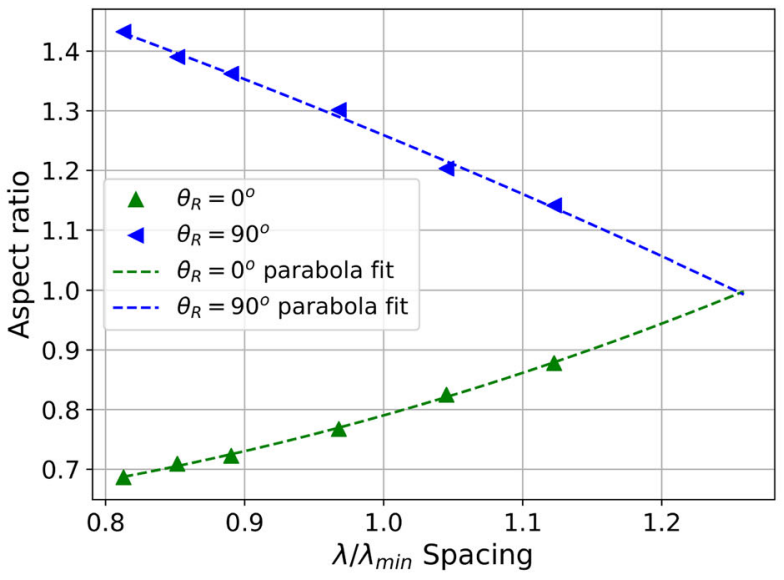

(d)

Fig. 5- ( $a$ through $c$ ) Top view of the solid-liquid interface for a spacing corresponding to $\lambda=69.6\left(\right.$ i.e., $1.09 \lambda_{\min }^{\text {iso }}$ or $\left.1.12 \lambda_{\min }^{\text {aniso }}\right)$, red $=$ Sn phase and blue $=\mathrm{Zn}$ phase for isotropic, $\theta_{\mathrm{R}}=0 \mathrm{deg}$ and $90 \mathrm{deg}$, respectively. No transition from the hexagonal arrangement of $\mathrm{Zn}$ phase is observed. (d) Aspect ratio of the cross-section of the steady-state anisotropic rods, calculated as the ratio of horizontal axis to vertical axis of the elliptical cross-section of the rod. Spacing is normalized with $\lambda_{\min }^{\text {aniso }}$ (Color figure online).

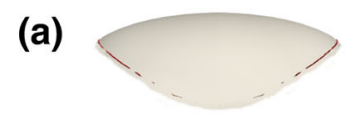

(b)
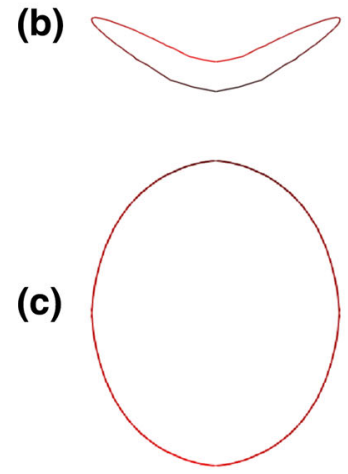

$$
\begin{aligned}
\lambda=52.8 & =0.85 \lambda_{\min }^{\text {aniso }} \\
\theta_{R} & =0^{0}
\end{aligned}
$$
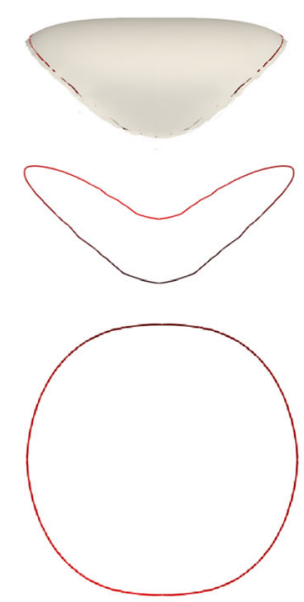
$\lambda=69.6=1.12 \lambda_{\min }^{\text {aniso }}$
$\theta_{R}=0^{0}$

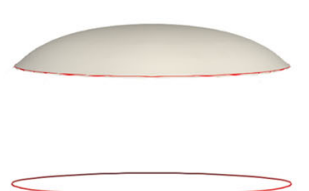

†

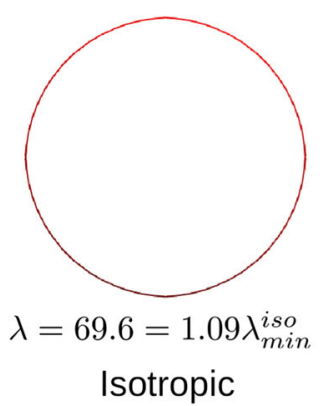

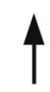

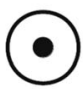

Growth

Direction

Fig. 6-(a) Solid-liquid interface corresponding to the rod phase for two different spacings $(\lambda=52.8$ in the left column and $\lambda=69.6$ in the center and right columns) and a comparison with the shape for the case of isotropic interfacial energy (right column). (b) and $(c)$ Comparisons of the triple lines in two different views. The growth direction for image rows $(a, b, c)$ is shown in the last column.

directions. The nature of the bifurcated shapes and the critical spacing depends on the domain and the volume fractions. However, the shape changes described previously in the presence of solid-solid interfacial energy anisotropy are different from the isotropic case as these occur continuously across the entire range of spacings where stable rod morphologies exist and do not arise as a result of a bifurcation beyond a critical spacing. 


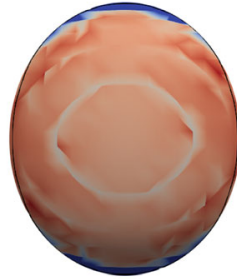

(a)
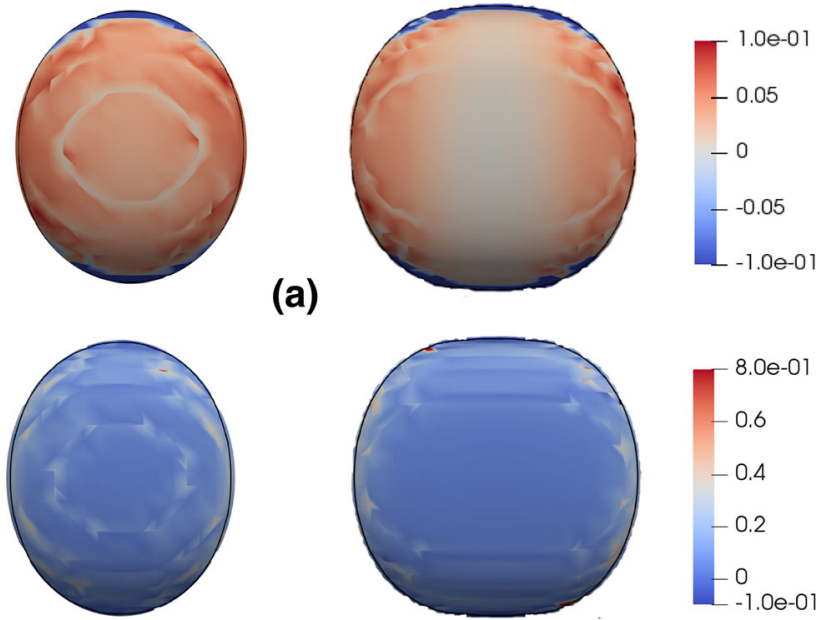

$$
\begin{gathered}
\lambda=0.85 \lambda_{\min }^{\text {aniso }} \\
\theta_{R}=0^{0}
\end{gathered}
$$

(b)

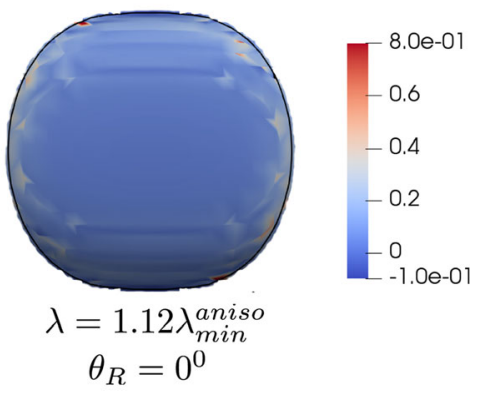

Fig. 7-Plots of the principal curvatures superimposed on the solid-liquid interface of the $\operatorname{rod} \kappa_{1}($ row $a)$ and $\kappa_{2}($ row $b)$ for $\lambda=$ $0.85 \lambda_{\text {min }}^{\text {aniso }}$ and $\lambda=1.12 \lambda_{\text {min }}^{\text {aniso }}$ for $\theta_{\mathrm{R}}=0 \mathrm{deg}$. Here $\kappa_{1}$ and $\kappa_{2}$ are calculated at each point of the rod-liquid interface as: $\kappa_{1}=$ $H-\sqrt{H^{2}-K}$ and $\kappa_{2}=H+\sqrt{H^{2}-K}$ where $H$ is the mean curvature and $K$ is the Gaussian curvature.

\section{Branching and morphology transition: large} spacings

We now shift our attention towards spacings larger than the minimum undercooling spacing that are amenable to branching instabilities (splitting of rod into two). Figure 8(1 and 3) (and also in the supplementary video 'Figure S1', refer to Electronic Supplementary Material) depicts the time sequence of morphology evolution for the two orientations of the solid-solid interfacial energy anisotropy $\theta_{\mathrm{R}}=0 \mathrm{deg}$ and $90 \mathrm{deg}$, with respect to the chosen hexagonal rod configuration. For a spacing corresponding to $\lambda=93.6$ (i.e., $1.46 \lambda_{\text {min }}^{\text {iso }}$ or $1.51 \lambda_{\min }^{\text {aniso }}$ ), in the isotropic case, the final morphology depends upon the initial rod shape (discussed later in this section), whereas for the anisotropic case, there is a morphological transition to a different arrangement depending only on the orientation $\theta_{\mathrm{R}}$. The branching instabilities occur as this spacing is beyond the stability limit, i.e., in the diffusion-dominated branch of the $\Delta T-$ $\lambda$ curve. Thus the system tends to reduce the spacing by branching of the rods, in order to accelerate diffusion and consequently decrease the overall interfacial undercooling. In order to understand the dynamics of this transition from the initial hexagonal rod morphology, we take a closer look at the temperature profile at the solid-liquid interface. From Figure 8(2 and 4), we note that the higher undercooling regions of the solid-liquid interface also correspond to the locations where the solid-solid interfacial energies are larger. As the $\mathrm{Zn}$ rod elongates in Figures 8(b) through (d), the interfacial area increases. This elongation happens such that the solid-solid interfaces with higher energy increase in area, which seems contrary to the notion that the system should always try to decrease its average undercooling.
However, this elongation is followed by branching of the $\mathrm{Zn}$ rod in Figure 8(e) (splitting of a rod into two rods in the top view corresponds to a branching in 3D), such that newly created interfaces are of the least energy. Due to this directional branching determined by anisotropy orientation, two distinct morphologies emerge, one is a transient arrangement for $\theta_{\mathrm{R}}=0 \mathrm{deg}$, and the other is a rectangular arrangement for $\theta_{\mathrm{R}}=90 \mathrm{deg}$. While in the case of $\theta_{\mathrm{R}}=90 \mathrm{deg}$, a steady state is reached, whereas for $\theta_{\mathrm{R}}=0 \mathrm{deg}$, microstructural evolution continues with the individual rods coming closer and aligning in a straight line, as highlighted in Figure 8(f1). Subsequently, they merge to form lamellae in Figures 8(g1) and (h1). This approach of individual rods towards each other only in the case of $\theta_{\mathrm{R}}=0$ deg can possibly be explained by the proximity of the regions corresponding to higher interfacial undercooling. After branching in Figure $8(\mathrm{e} 2)$, the interfaces with a higher undercooling are closer for $\theta_{\mathrm{R}}=0 \mathrm{deg}$ (approximately half of the original rod spacing) and there exists a topological pathway by which the regions of higher undercooling can first come towards each other, by a lateral translation of the rods and after alignment, lengthening and merging along the higher undercooling directions. The configuration with $\theta_{\mathrm{R}}=90 \mathrm{deg}$ also has the higher undercooling regions of the rods separated by the shorter distance of $\lambda / 2$, however, no mechanism exists for the direct merging of these regions through the growth of the rods towards each other in the $Y$ direction. For the case of $\theta_{\mathrm{R}}=0 \mathrm{deg}$, the higher undercooling interface regions are eliminated by alignment of the $\mathrm{Zn}$ rods, where they subsequently merge to form lamellae. Thus, the average interfacial undercooling shows a sharp dip as the morphology transitions to lamellar around a time of 20 (all time values represented in the paper are scaled by $\left.v / \lambda_{\min }\right)$, as shown in Figure 9(a). Once a steady-state morphology is reached (after time $=25$ for $\theta_{\mathrm{R}}=0$ deg and time $=9$ for $\theta_{\mathrm{R}}=$ $90 \mathrm{deg}$ ), the interfacial velocity and undercooling do not vary with time in Figures 9(a) and (b) and thereby these are steady-state configurations for these spacings.

The above conclusion can very well be observed from the average $\Delta T-\lambda$ plot in Figure $9(\mathrm{c})$. The $\lambda$ axis represents the initial spacing at the start of the simulation. The curves correspond to fitting of simulation points according to the equations of the type $\Delta T=$ $k_{1} v \lambda+k_{2} / \lambda$. The lamellar morphology for the isotropic case has a higher average undercooling than that of the corresponding rod microstructure, which is expected when the volume fraction of the minority phase is far lower. For spacings outside the stability window (two of them are shown), the morphologies which transform to rectangular and lamellar configurations have a lower average interfacial undercooling. As the rod-to-lamellar transition occurs, it leads to a lamellar configuration where the solid-solid interfaces are oriented such that the solid-solid interfacial energies are lower $\left(\gamma_{\mathrm{Sn}-\mathrm{Zn}}=1.022\right)$ than for the case of the simulation with the lamellar morphology with isotropic interfacial energies $\left(\gamma_{\mathrm{Sn}-\mathrm{Zn}}=1.46\right)$. Accordingly, this lamellar arrangement formed upon transformation from the rod morphology has a lower undercooling. The 


$$
\theta_{R}=0^{0}
$$

\section{Solid-liquid interface Temperature}

(a)

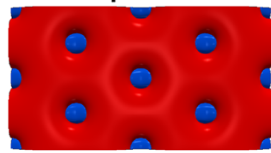

(b)

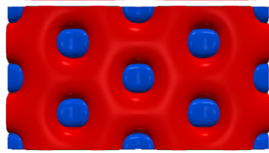

(c)

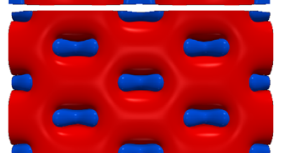

(d)

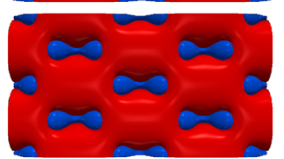

(e)

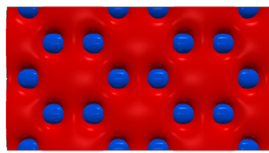

(f)

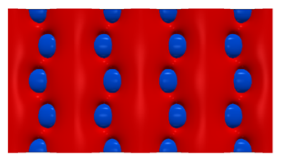

(g)

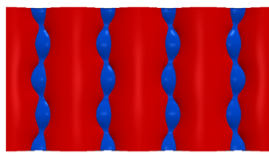

(h)

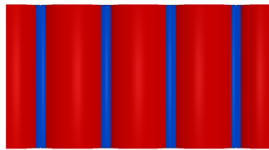

1
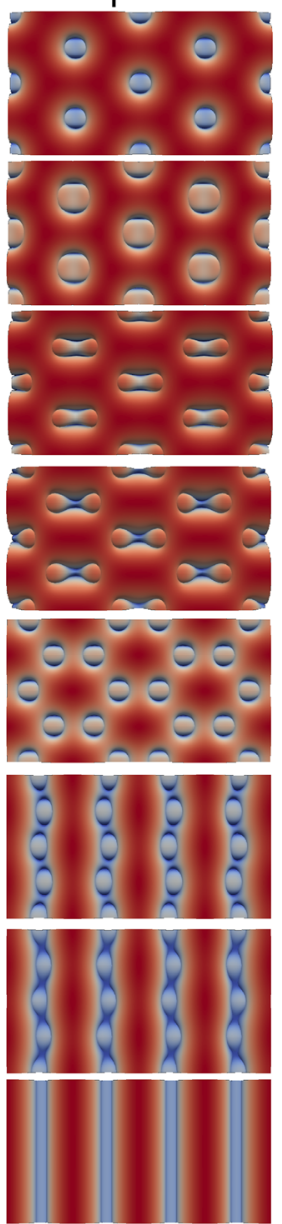

2
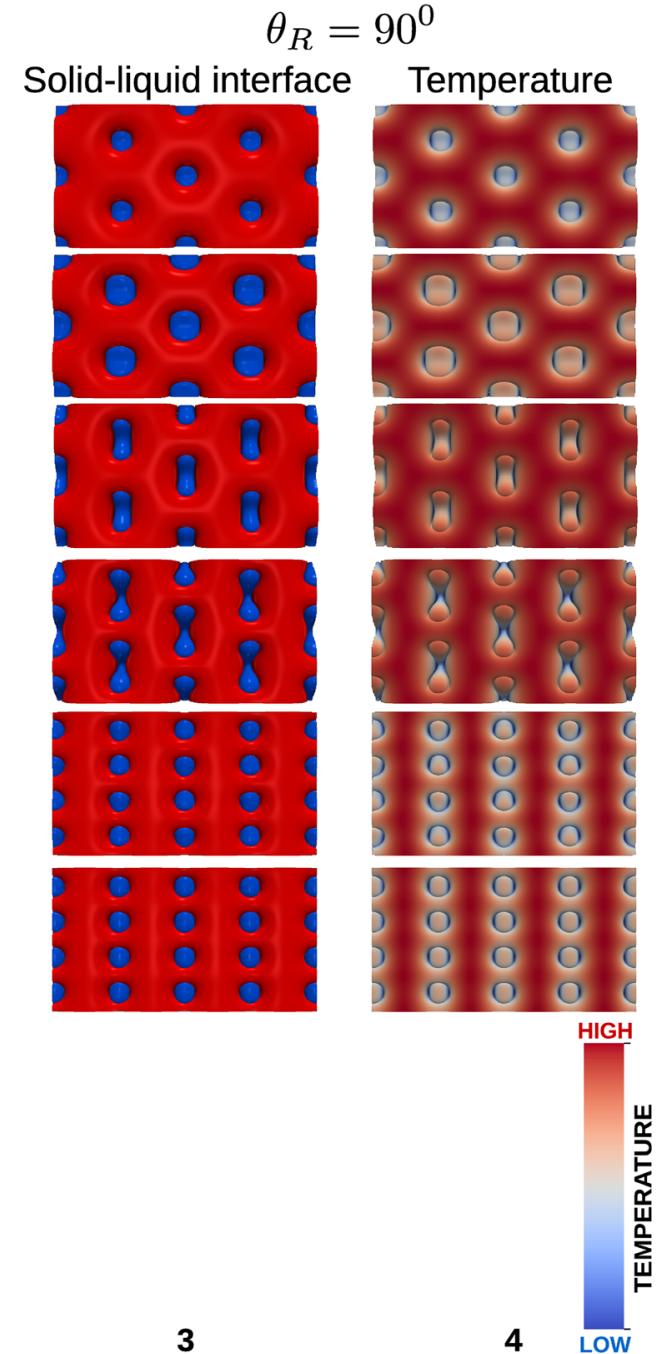

Fig. 8-Morphology evolution with time increasing from row $(a)$ through $(h)$, where columns 1 and 3 are the top views of the solid-liquid interface, columns 2 and 4 depict the temperature field superimposed on the solid-liquid interface (coloring as per the temperature legend, red $=$ lower undercooling and blue $=$ higher undercooling). Column 1 shows hexagonal rod-to-lamellar transition, column 3 shows a transition to a rectangular arrangement for two different orientations of the solid-solid interfacial energy anisotropy function (Color figure online).

rectangular arrangement has two possible spacings, one $(\lambda / 2)$ in the vertical direction and the second $(\sqrt{3} \lambda / 2)$ in the lateral direction, whereby it is a different arrangement which corresponds to none of the undercooling vs spacing relations of either the rod or the lamella.

It is worth pointing out that the spacing beyond which the hexagonal configuration becomes unstable to branching instabilities $\left(\lambda / \lambda_{\min }^{\text {aniso }} \approx 1.33\right)$ is approximately just beyond the point where the aspect ratio of the cross-section of the rod becomes nearly circular $\left(\lambda / \lambda_{\min }^{\text {aniso }}=1.26\right)$ as in Figure $5(\mathrm{~d})$. The branching of the rods occurs by elongation in the directions corresponding to the lower interfacial energies and narrowing of the rods along the regions of higher undercooling (or higher solid-solid interface energies) where the matrix phase invades the rod phase leading to splitting. Branching also occurs in isotropic situations giving rise to arrangements that are similar (for $\lambda / \lambda_{\min }^{\text {iso }} \geq 1.46$ ); however, there is no transition to a lamellar state.
Figure 10 shows arrangements that are derived post-branching for an initial spacing of $\lambda=93.6$, corresponding to $\lambda / \lambda_{\min }^{\text {iso }}=1.46$, starting with two different initial rod shapes: horizontally elongated ellipse in column 1 whereas vertically elongated ellipse in column 2. The results are somewhat different to those reported in Reference 68 as we do not find broken lamellar states in our simulations, but rather a connected rod morphology. Additionally, we find a change in the arrangement of the rods, whereupon starting with an ellipse that is elongated vertically we derive a rectangular arrangement of nearly touching rods. In terms of the differences in the branching behavior between the isotropic and the anisotropic cases, firstly the critical spacing where branching events occur in isotropic situations shifts to larger values of $\lambda / \lambda_{\min }^{\text {iso }}=1.46$ in comparison to the anisotropic case $\left(\lambda / \lambda_{\min }^{\text {anis }} \approx 1.33\right)$. Moreover, the nature of branching is non-directional for the case of isotropic interfacial energies, while it is biased in the presence of 


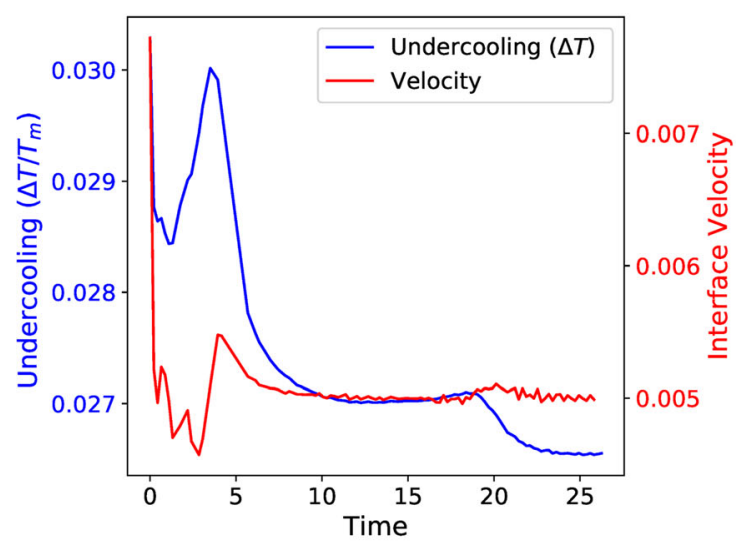

(a)

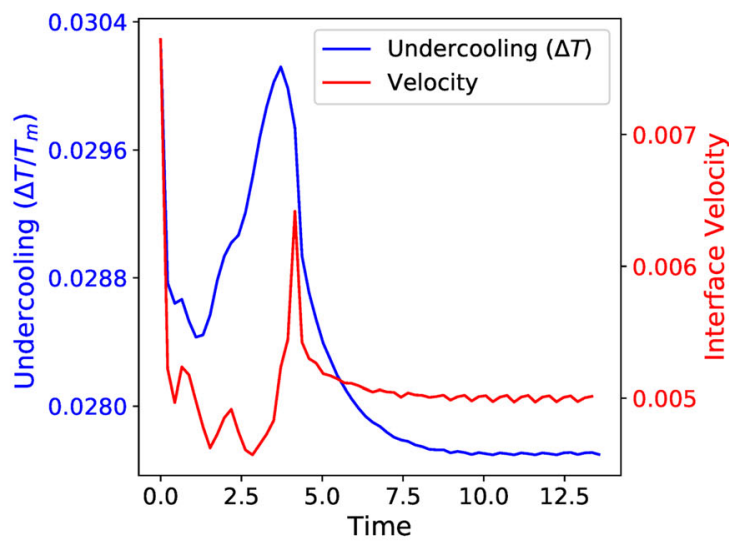

(b)

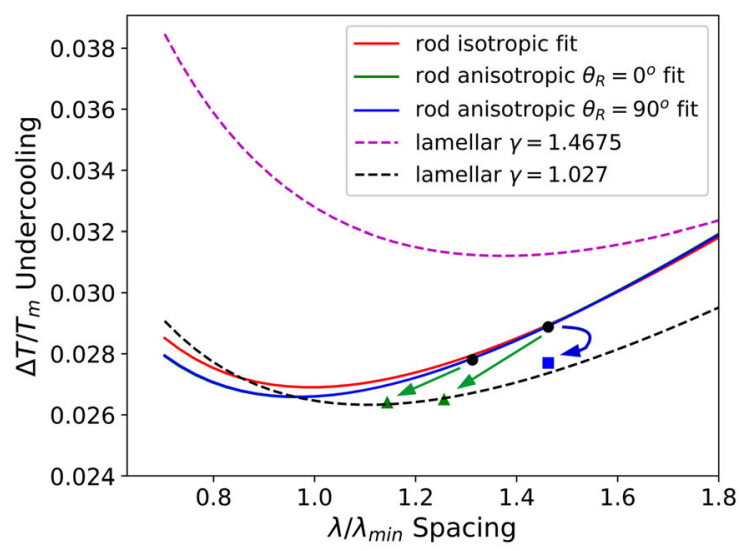

(c)

Fig. 9-Undercooling $(\Delta T)$ and velocity with time (time normalized with $\left.v / \lambda_{\min }\right):(a) \theta_{\mathrm{R}}=0$ deg and $(b) \quad \theta_{\mathrm{R}}=90$ deg. Reduction in undercooling $(\Delta T)$ around time $=20$ in (a) is due to transition to a lamellar morphology. A steady-state arrangement with a constant $\Delta T$ is attained towards the end of simulation in both (a) and $(b)$. (c) Transformed lamellar states (green triangle, $\theta_{\mathrm{R}}=0$ deg) and rectangular arrangements (blue square, $\theta_{\mathrm{R}}=90 \mathrm{deg}$ ) have a lower undercooling than the hexagonal (black circle) configuration they started with. Here, the spacing is scaled with $\lambda_{\min }=\lambda_{\min }^{\text {iso }}$ (Color figure online).

(a)

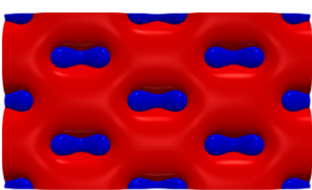

(b)

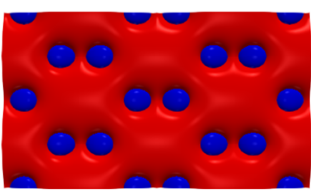

1
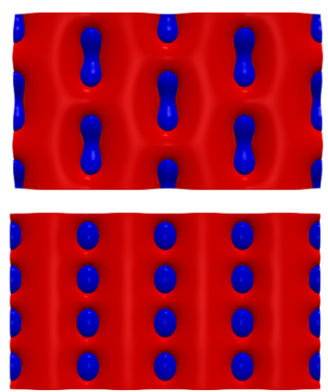

2

Fig. 10-Arrangement of rods (a) during branching and (b) steady state for two different starting situations (column 1 is a horizontally elongated ellipse and column 2 is a vertically elongated ellipse) for isotropic solid-solid interfacial energies. In both cases, we observe incomplete merging events leading to connected elongated rod morphologies arranged in either a hexagonal or in a rectangular lattice.

anisotropy in the solid-solid interfacial energy. This is because anisotropy provides a natural perturbation to the solid-liquid interface making it amenable for the invasion of the rod phase in the regions of higher undercooling. Thus, while in isotropic situations, branching in all directions can occur, where the mode of branching depends on the starting conditions or the departure from the isotropic hexagonal arrangement, ${ }^{[68]}$ in the presence of anisotropy, the direction of branching, as well as the final state of the microstructure depends strongly on the orientation of the anisotropy. Additionally, while no lamellar states are derived in the isotropic case whereas in the presence of anisotropy, the merging of rods post-branching events leads to the elimination of higher undercooling regions leading to the formation of lamellae. The merging of rods occurs, following the evolution of the rods towards each other, where given that the interfacial undercooling varies along the triple line, the rod evolution occurs such that the regions of higher undercooling approach each other (see Figures $8(\mathrm{e})$ through $(\mathrm{g}))$. Therefore, it is apparent that it is this difference in undercooling along the triple line 
$\theta_{R}=0^{0}$

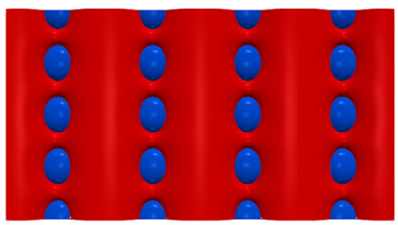

$\theta_{R}=90^{\circ}$

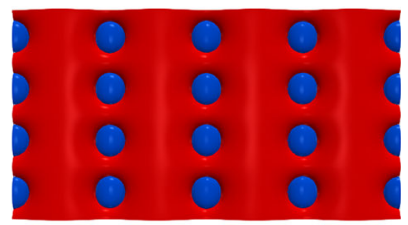

(a)

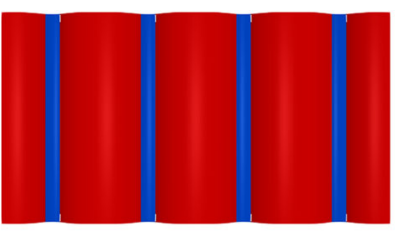

1

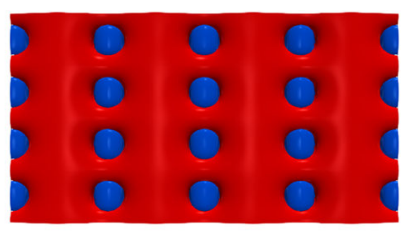

2 (b)

Fig. 11-Steady-state solid-liquid interface, (a) $\delta_{\mathrm{Sn}-\mathrm{Zn}}=0.15, \quad(b)$ $\delta_{\mathrm{Sn}-\mathrm{Zn}}=0.30$. For higher $\delta_{\mathrm{Sn}-\mathrm{Zn}}$ we find alignment of the rods by lateral translation and then merging, whereas for lower anisotropy strength $\delta_{\mathrm{Sn}-\mathrm{Zn}}$, only alignment of the rods occurs after branching but no merging. Higher anisotropy strengths and appropriate orientations of the solid-solid interfacial energy lead to a lamellar morphology as in b1 by merging of solid-solid interfaces, in the constrained simulations.

that provides a natural transition mechanism to a lamella. Since the difference in undercooling along the triple line is a function of the anisotropy, we expect the possibility of transformation to a lamella to depend on the strength of anisotropy. This can be appreciated from the results depicted in Figure 11 where a rectangular arrangement of elongated rods is derived for an anisotropy strength that is $\delta_{\alpha \beta}=0.15, \theta_{\mathrm{R}}=0 \mathrm{deg}$ that is half of the previously utilized value of $\delta_{\alpha \beta}=0.3, \theta_{\mathrm{R}}=$ 0 deg where complete merging occurs. We note that here as well the rods branch in a manner that leads to the invasion of the majority phase in the higher undercooling regions of the rod phase and a similar gradual alignment of the rods as depicted in Figure 8 and also in the supplementary video 'Figure S1' (refer to Electronic Supplementary Material), leading to the formation of a rectangular array, but no lamella formation. Therefore, we can draw an inference that with increasing strength of anisotropy, the possibility of a transition from a rod to a lamellar arrangement increases. While the constrained simulations reveal the influence of anisotropy on the rod shapes, the eventual evolution of the microstructures are constrained by the imposed domain boundary conditions as revealed in the simulations for the orientation $\theta_{\mathrm{R}}=90 \mathrm{deg}$, where although branching occurs, they do not merge leading to the lamellae formation. Thus, the steady-state morphology achieved under the imposed domain constraints might not reflect the final steady state possible in an extended simulation with more spatial degrees of freedom to rearrange. However, these results show us exclusively the influence of interfacial energy anisotropy on the rearrangement dynamics.

\section{B. Extended Simulations}

Having studied the effect of anisotropic interfaces on constrained simulation domains, we now explore the influence of anisotropy on microstructural evolution in large simulation domains comparable to experimental conditions. Thus, we choose a simulation box of cross-section $\left(8 \lambda_{\min }\right)^{2}$ and initialize with a random arrangement of $\mathrm{Zn}$ phase bricks in the Sn matrix. Simulations are performed for two cases: isotropic and anisotropic solid-solid interface energies, where we initialize both the isotropic and the anisotropic simulations with the same random arrangement. In the anisotropic case, the orientation of 2 -fold interface energy anisotropy function is $\theta_{\mathrm{R}}=0 \mathrm{deg}$ for all the $\mathrm{Sn}-\mathrm{Zn}$ interfaces, thereby replicating conditions of growth in a single eutectic grain. The strength of anisotropy is $\delta_{\mathrm{Sn}-\mathrm{Zn}}=0.3$. For this strength of anisotropy, we expect the formation of a lamellar morphology as observed in the constrained simulations.

\section{Morphology evolution with time}

The isotropic case reaches a steady state as the interfacial undercooling and velocity become nearly constant in Figure 12(a), which is not observed in the anisotropic case in Figure 12(b). The average undercooling still decreases in the anisotropic case as the morphology transitions from random $\rightarrow$ rectangular $\rightarrow$ aligned broken lamellar in Figure 13 (right column) (also in the supplementary video 'Figure S2', refer to Electronic Supplementary Material). In order to analyze the lengthening dynamics of anisotropic lamella, we plot the average fragment length and the length of an individual fragment against simulation time in Figure 14. The lamella fragment is not always straight, and hence, we have calculated the lamella length considering its actual geometry. First we consider a transverse section ( $X Y$ plane) below the solid-liquid interface where only the solid phases are present. Thereafter, we identify the different lamellar/rod fragments using the Hoshen-Kopelman algorithm ${ }^{[71]}$ as shown in Figure 14(a). For each lamella fragment in this transverse plane, we construct a central line (curve) passing through the fragment in the following way: for each $y$ coordinate, the $x$ coordinate corresponding to the centroid of the lamella fragment is calculated, whereupon joining these points, we derive the central line of each fragment (see the central black line in Figure 14(a)). The arc length of this central line is the length of the lamella fragment which is computed by the cumulative sum of the lengths of the discrete line segments forming the central line and this accounts for cases where the fragments are curved. In Figure 14(d), an individual lamella elongates linearly with time, interspersed with jumps in the length when merging with a neighboring fragment occurs. Moreover, for this particular lamella fragment, we track the triple-point region with time, along a longitudinal section ( $Y Z$ plane) (see Figure 14(b)) intersecting the leading edge of the lengthening lamellar fragment at its 'tip', i.e., the $x$ coordinate of the $Y Z$ plane is chosen such that the plane contains the highest $y$ coordinate of the particular 


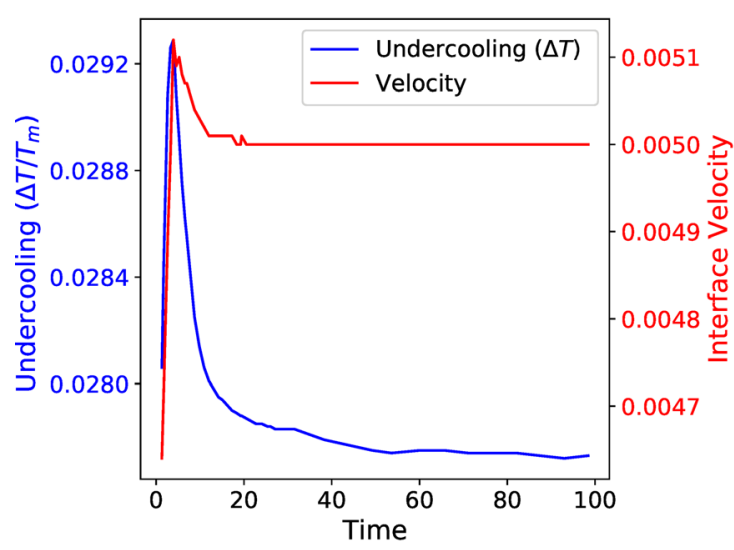

(a)

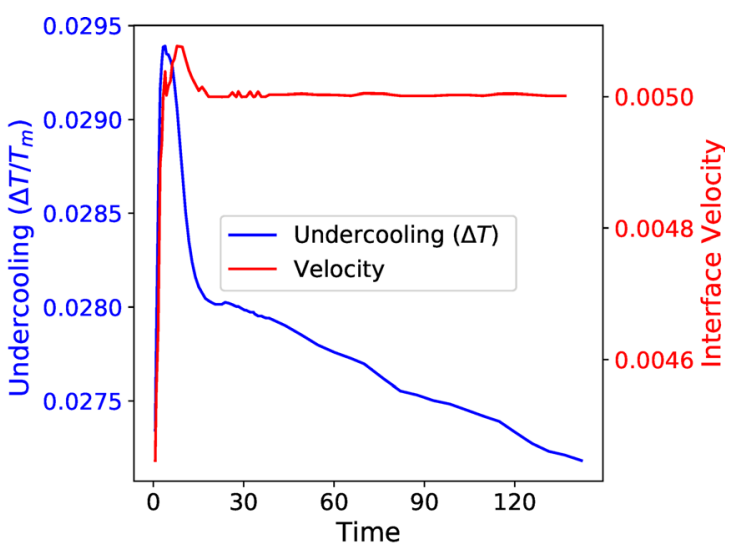

(b)

Fig. 12-Interface undercooling $(\Delta T)$ and velocity $v s$ time (time normalized with $\left.v / \lambda_{\min }\right)$ for $(a)$ isotropic and $(b)$ anisotropic interfacial energy. (a) Attains a steady state, (b) moving towards steady state with undercooling still decreasing.

lamella (see Figure 14(b), where the white line is the longitudinal plane, which cuts the lamella tip inside the black ellipse). The triple-point regions are displayed in Figure 14(e), where we observe that the solid-solid interface has a tilt with respect to the imposed direction of the thermal gradient. We have measured the magnitude of this tilt angle (calculated from the slope of the solid-solid interface line in Figure 14(e)) as a function of time between two merging events and plotted in Figure 14(f). Correlating the tilt angle measurements with the length of a single lamella fragment as in Figure 14(d), we see that just before a merging event, the tilt of the solid-solid interface increases and thereafter reduces to a minimum value before it starts to increase again when it approaches a second fragment before merging. The value of the minimum angle of tilt is approximately the same between any two merging events and this value is approximately $(\approx 1.27 \mathrm{deg})$ from the vertical temperature gradient direction. The tangent of the tilt angle is nearly equal to the ratio of the lamella lengthening velocity $\left(\approx 1.08 \times 10^{-4}\right)$ to the imposed directional solidification velocity $(0.005)$ thereby indicating that locally the growth of the lamellae fragment is at an angle to the imposed thermal gradient direction. Thus, the lamella also traverses in the $Y$ direction with the $Y$ component of the growth velocity. Therefore, the lengthening dynamics of the lamella between two merging events is proportional to the imposed directional solidification velocity as well as the tilt of the solid-solid interface with respect to the growth direction. We expect this tilt of the tri-junction that leads to the invasion of the phase with the lower volume fraction while also resulting in merging events and elongation of lamellar fragments, to be a function of the strength of solid-solid interfacial energy anisotropy, the imposed velocity, as well as the local spacing and arrangement of the lamella fragments/rods in the extended simulation domain. The influence of the local conditions is evident in the supplementary video 'Figure S2' (refer to Electronic Supplementary Material), wherein there exist fragments that do not monotonically increase in length and there are times for which the length of a given fragment also reduces. However, the average length of the lamella fragments increases monotonically with time.

Since the merging events occur along the directions corresponding to the higher solid-solid interfacial energies, a natural biasing of the microstructure where lamellae globally assume a common solid-solid interface orientation and thereby a uniform interfacial energy is expected. The alignment of the lamellae is revealed in the two-point spatial correlation map (see Figure 15 (right column)) which also indicates the gradual lengthening of lamellae fragments with time as derived from the lengthening of the maximum intensity spot at the center. Additionally, the correlation depicts the development of a uniform lamellar spacing with time as highlighted by the separation of the linear streaks in the spatial correlation plot. Conversely, the spatial correlation of the isotropic case (see Figure 15 (left column)) highlights the development of the hexagonal symmetry in the rod arrangement with the time of solidification. While there is globally a tendency towards alignment of the lamella fragments, during merging in the presence of solid-solid interfacial energy anisotropy, there do occur small changes in the solid-solid interface orientation during growth. This is to be expected as locally the arrangement of the lamella fragments/rods is not perfectly in position for merging with the same given solid-solid interface orientation. Deriving from the results of the constrained simulations, where the rods approach each other such that the higher undercooling interface regions come closer by motion in the transverse plane, the lamellae fragments in the extended simulations are similarly expected to change growth directions during the merging events, leading to small changes in the solid-solid interface orientations. The fact that the merging events are globally correlated through the diffusion field in the liquid and not just a function of the local interfacial conditions is revealed in the development of a uniform lamellar spacing during the merging events. 


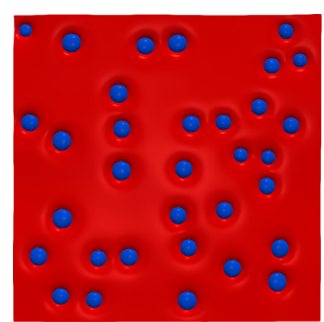

(a)

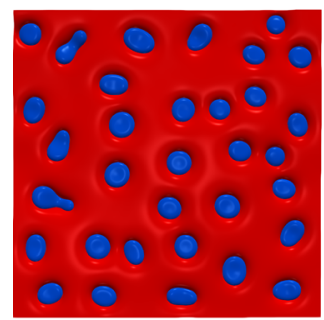

(c)

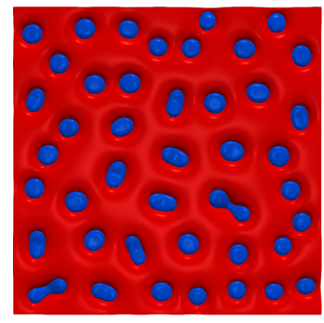

(e)

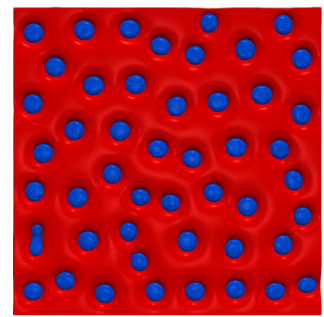

(g)

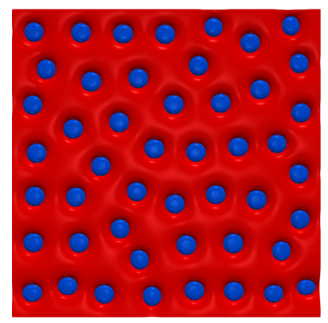

(i)

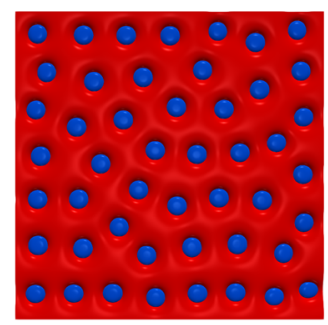

(k)

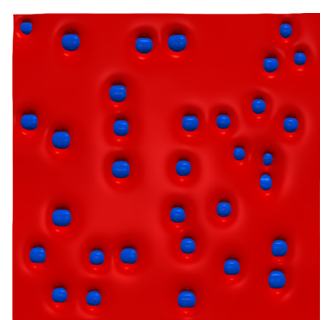

(b)

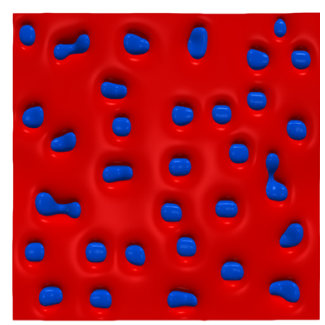

(d)

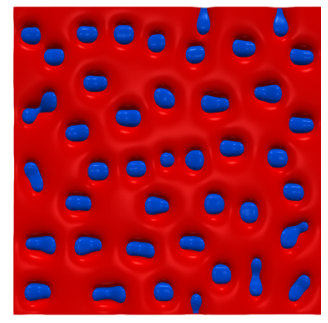

(f)

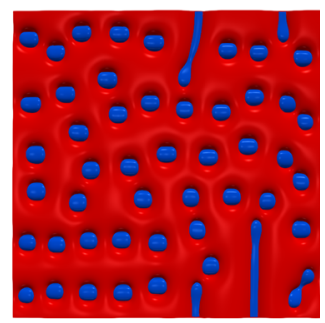

(h)

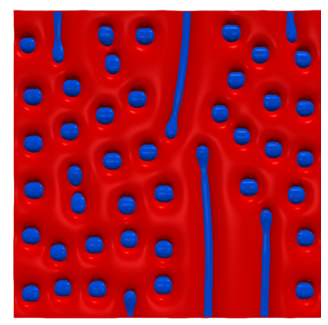

(j)

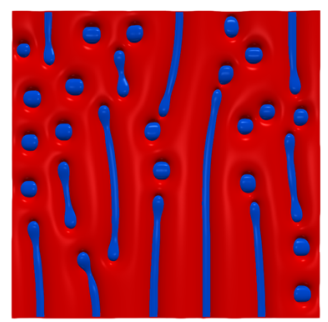

(I)
4Fig. 13- ( $a$ through $l$ ) Top views of the solid-liquid interface during morphological evolution, where the left column is for isotropic interfacial energies (time increasing from 0 to 100), while the right column is for anisotropic Sn-Zn interfacial energies (time increasing from 0 to 142). Starting from the same random morphology, the isotropic case gradually transitions towards a hexagonal configuration, while the anisotropic one transitions towards a rectangular arrangement of $\mathrm{Zn}$ rods and eventually to a broken lamellar morphology. The simulation time is normalized with $v / \lambda_{\min }$.

\section{Comparison with experiment}

The results from our simulations suggest that the presence of anisotropy in the solid-solid interfacial anisotropy provides mechanisms for the formation of lamellae microstructures even when the volume fractions of the minority phase is below the critical value for rod-lamella transition in isotropic systems. The merging of lamellar fragments during solidification is observed in our experimental investigations of this alloy (that are reported elsewhere). Figure 16 reveals the gradual lengthening of the average lamellar fragment length with time. While we do not observe actual merging events, we see that the lengthening of the lamellar fragments is biased in a given direction in this eutectic grain. Since our experimental investigations indicate the presence of solid-solid interfacial anisotropy(that is reported elsewhere), the combination of the morphological changes as seen in Figure 16 leading to the lamellae formation in the experiments and the simulation results that reveal the mechanisms by which such transitions occur provides strong evidence that the presence of solid-solid interfacial energy anisotropy leads to the formation of lamellar microstructures even in these systems where the minority fractions are low. We note that our conclusions are notwithstanding the simplified assumptions with respect to the choice of the form of anisotropy in the solid-solid interfacial energy where we have applied the simplest form which satisfies the symmetry of the experimentally observed solid-solid interfaces. From the envelope of the solid-solid interfaces in Figure 16, a 2-fold symmetry is expected for the solid-solid interfacial energies for orientations where the normal to the solid-solid interface lies in the transverse plane. However, the actual 3D Wulff shape corresponding to this system might indeed be more complicated. While we do not address the additional contributions that might be attributed to the symmetry features of the real anisotropy function, the modeling effort does clarify that the presence of solid-solid interfacial anisotropy even in its simplest form leads to the formation of lamellar structures. This evidence that we have presented has therefore implications for several such alloys where lamellar structures are observed. However, the measurement of the actual form of the anisotropy of the solid-solid interfaces and its utilization in a phase-field simulation remains a scope for future, experimentally informed theoretical and numerical work. 


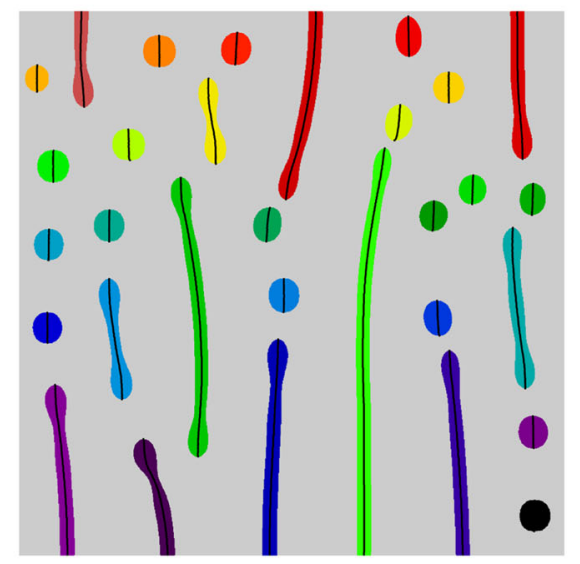

(a)

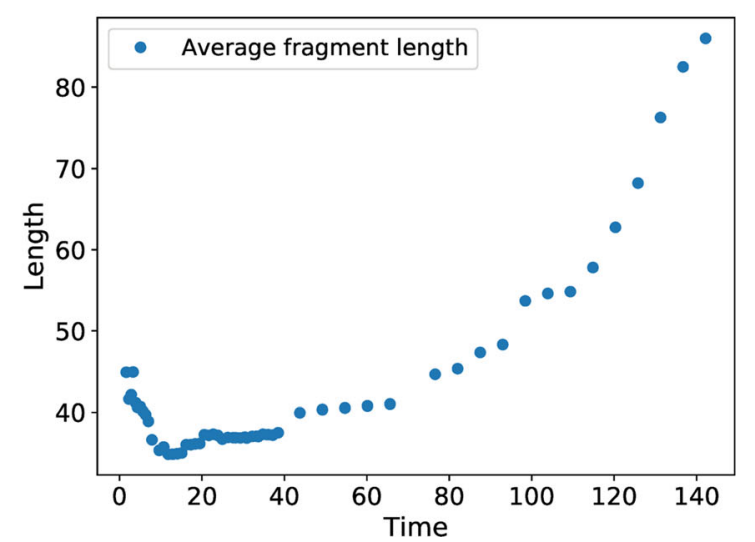

(c)

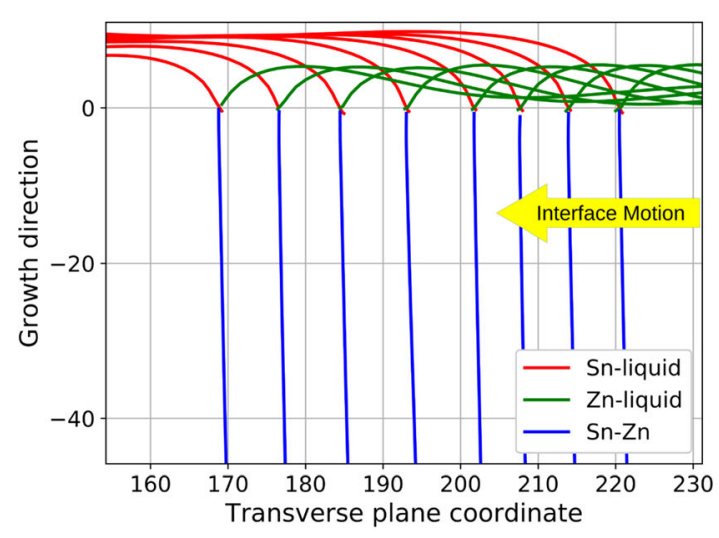

(e)

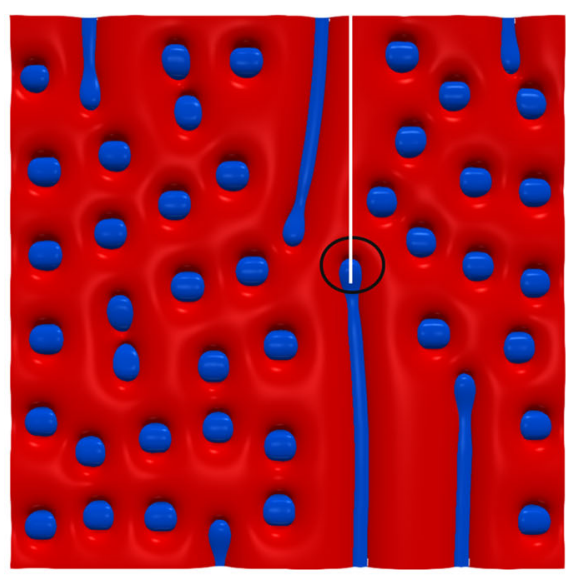

(b)

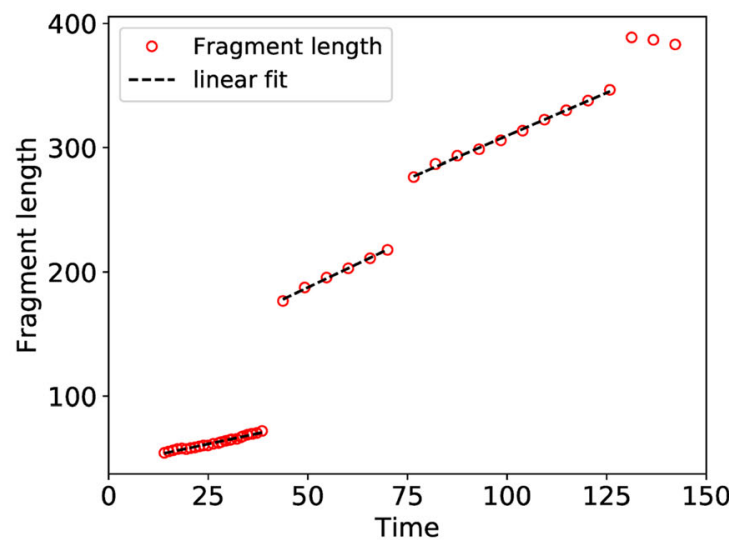

(d)

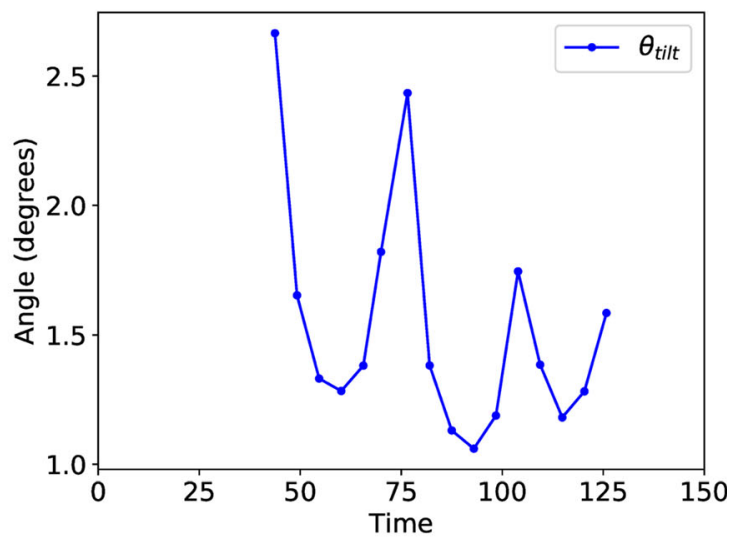

(f)

Fig. 14-(a) Transverse $(X Y)$ plane for the fragment length calculation. The black line inside each fragment shows the central line used for calculation of the lamellar length. $(b)$ The white line is the longitudinal $(Y Z)$ plane intersecting the lengthening fragment at its tip depicted inside the black ellipse. (c) Average lamella length with time. (d) Length of an individual lamella fragment grows linearly with time (fit with black dashed line), interspersed with jumps that depict merging events (time normalized with $\left.v / \lambda_{\min }\right)$. (e) Longitudinal cut-section of all interfaces near the triple line region along the leading interface front for the same lamella in (b). The lamella elongates towards the left with increasing time from 82 to 121 . The solid-solid interface is tilted from the vertical temperature gradient direction. $(f)$ Plot of the tilt angle w.r.t. to the imposed thermal gradient direction of the solid-solid interface with time. 

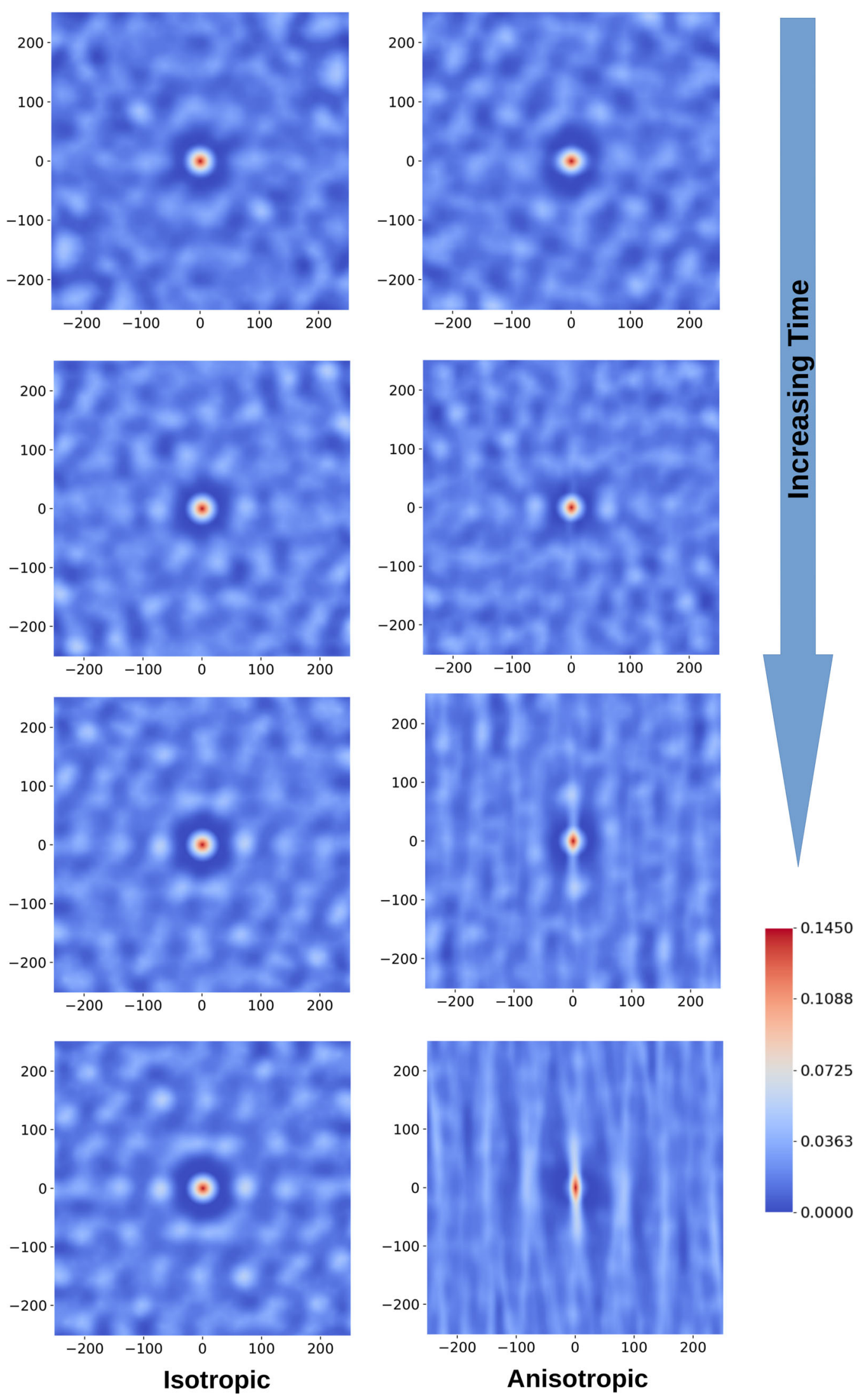

Fig. 15-2-Point self-correlation of the $\mathrm{Zn}$ phase at different simulation times, the left column is for isotropic interfacial energies, the right column is for anisotropic $\mathrm{Sn}-\mathrm{Zn}$ interfacial energies. A hexagonal periodicity emerges in the isotropic case with time, whereas the anisotropic tends towards a broken lamellar pattern. 

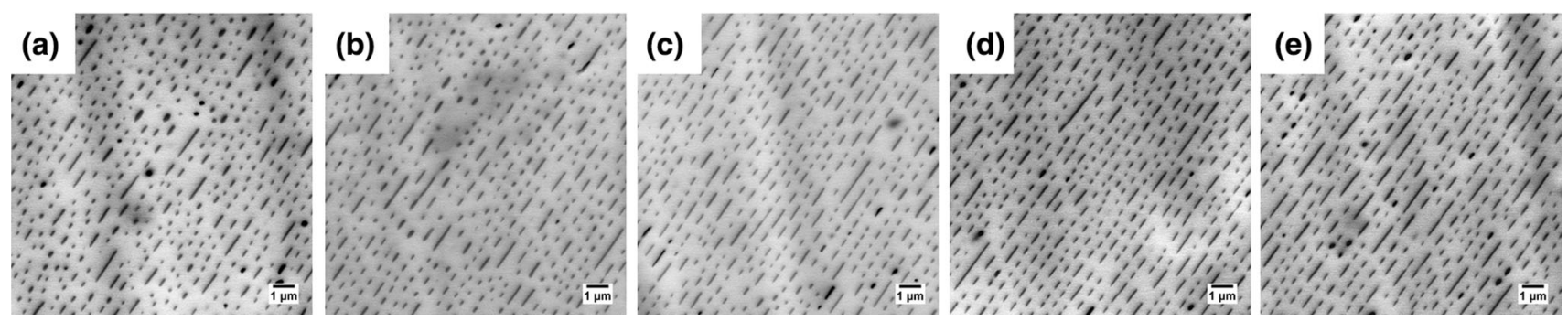

Fig. 16-Images captured using a scanning electron microscope (SEM), which are taken at equally spaced transverse sections along the growth direction, following the same eutectic grain of the sample solidified at $50 \mu \mathrm{m} / \mathrm{s}$. (a) is $20 \mathrm{~mm}$ from the bottom, $(b)$ is $30 \mathrm{~mm}$ from the bottom, (c) is $40 \mathrm{~mm}$ from the bottom, $(d)$ is $50 \mathrm{~mm}$ from the bottom, and $(e)$ is $60 \mathrm{~mm}$ from the bottom. The images indicate a gradual lengthening of the average lamella fragment length as a function of time.

\section{CONCLUSIONS}

In this paper, we present evidence using phase-field simulations that the presence of anisotropy in the solid-solid interfacial energy leads to the formation of the lamellar morphology even in systems where the minority phase fraction is low. Though we created a model system mimicking properties similar to those exhibited by the eutectic phases in the $\mathrm{Sn}-\mathrm{Zn}$ alloy, the particular observations regarding the influence of anisotropy are generic and are applicable to the coupled growth of any two-phase eutectic system. The rod-lamellar transition occurs via a branching and merging mechanism, wherein the rods first branch in order to reduce diffusion distances, while new solid-solid interfaces are produced. Thereafter, merging of rods occurs such that the higher energy orientations are eliminated and orientations that are retained correspond to lower energy and thereby also resulting in reduced average undercooling of the solid-liquid interface. We note that the first step of branching is common between both isotropic and anisotropic systems; however, the formation of lamellae by merging along specific orientations is observed only for the case where the solid-solid interfacial energies are anisotropic. While such merging events can certainly occur in isotropic situations with an increase in volume fractions, there is no strong basis to expect any biasing with respect to the formation of either lamellae or lamella fragments similar to what we find for the case with anisotropic solid-solid interfacial energies. Therefore, it is not surprising that the propensity for rods to merge and form lamellae depends on the strength of anisotropy, with a higher strength of anisotropy giving rise to a greater tendency for lamella formation. Additionally, we also note that the merging of lamella fragments proceeds via several invading lamellae-front where the properties of the tri-junction such as the tilt of the solid-solid interface during the invasion remains fairly constant for a particular lamella fragment for long periods of the invasion. We, however, expect the tilt of the solid-solid interface at the leading front of the invading lamella fragment to be a function of the velocity, local spacing, and the anisotropy strength of the solid-solid interfacial energy and thereby the lengthening dynamics must vary spatially as well as depend on the solidification velocity and the strength of anisotropy in the solid-solid interfacial energy. We find evidence of the lamella fragment lengthening process in our own experiments where the lengthening occurs along given crystallographic directions that are supported by the simulation results in this work. Therefore, using a combination of experiments and simulations, we are able to precisely determine the effect of anisotropic solid-solid interfacial energy on the resulting microstructure, particularly on the rod-to-lamellar transition in eutectic system, that was not quite clear until now.

\section{ACKNOWLEDGMENTS}

The authors would like to thank DST-SERB for funding through the Project (DSTO1679). SK would like to thank SERC and TUE-CMS, IISc, for providing access to high-performance computational resources, including the use of the SahasraT (Cray XC40) machine at SERC. The authors would also like to thank Prof. Mathis Plapp for insightful discussions during the course of the work.

\section{APPENDICES}

\section{A. NON-DIMENSIONALIZATION OF SIMULATION PARAMETERS}

The parameters used in the simulation are obtained through non-dimensionalization of the physical parameters. The procedure is enlisted below, where the asterisked values are defined as: $T^{*}=471.7, f^{*}=\frac{R T^{*}}{V_{\mathrm{m}}}$, $l^{*}=\frac{\sigma}{f^{*}}$ and $t^{*}=\frac{l^{* 2}}{D}$. Here, the values of molar volume $V_{\mathrm{m}}=1.6 \times 10^{-5} \mathrm{~m}^{3}$, interface energy $\sigma=0.104 \mathrm{~J} \mathrm{~m}^{-2}$ and diffusivity $D=3.5 \times 10^{-9} \mathrm{~m}^{2} \mathrm{~s}^{-1}$ are obtained from References 40,60. Dividing the dimensional parameters with the corresponding asterisked (*) variables gives their non-dimensional values. 


\section{B. NUMERICAL CONSISTENCY AND GRID RESOLUTION}

The simulations have been checked for numerical accuracy against different grid resolutions $(\mathrm{d} x)$ and interface widths as shown in Figure AI.

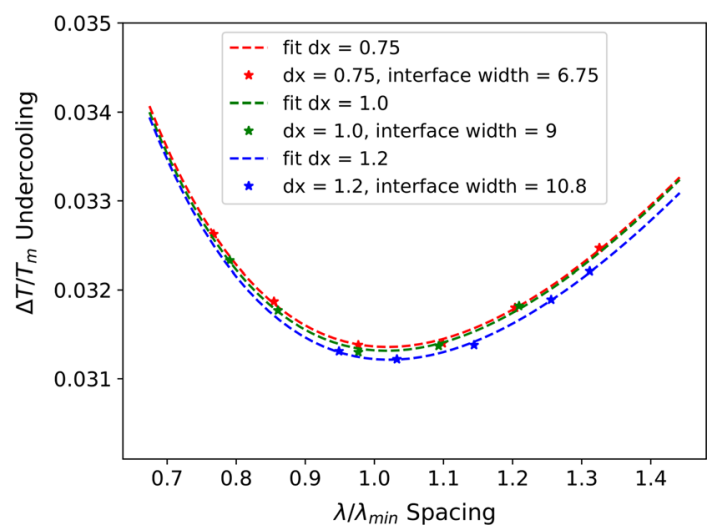

Fig. AI-Numerical consistency and grid resolution, checked with different interface widths.

\section{ELECTRONIC SUPPLEMENTARY MATERIAL}

The online version of this article (https://doi.org/10. 1007/s11661-020-05995-8) contains supplementary material, which is available to authorized users.

\section{REFERENCES}

1. W. Kurz and D.J. Fisher: Fundamentals of Solidification, Trans Tech Publications, Aedermannsdorf, 1986, vol. 1.

2. J.A. Dantzig and M. Rappaz: Solidification, EPFL Press, Lausanne, 2009.

3. G.A. Chadwick: Prog. Mater. Sci., 1963, vol. 12, pp. 99-182.

4. J. Hunt and K. Jackson: Trans. Metall. Soc. AIME, 1966, vol. 236, pp. 843-52.

5. R. Elliott: Eutectic Solidification Processing: Crystalline and Glassy Alloys, Elsevier, Amsterdam, 2013.

6. M. Croker, R. Fidler, and R. Smith: Proc. R. Soc. Lond. A, 1973, vol. 335 , pp. $15-37$.

7. K. Kassner and C. Misbah: Phys. Rev. A, 1991, vol. 44, p. 6513.

8. U. Hecht, L. Gránásy, T. Pusztai, B. Böttger, M. Apel V. Witusiewicz, L. Ratke, J. De Wilde, L. Froyen, D. Camel et al.: Mater. Sci. Eng. R, 2004, vol. 46, pp. 1-49.

9. A. Karma and M. Plapp: JOM, 2004, vol. 56, pp. 28-32.

10. H. Walker, S. Liu, J. Lee, and R. Trivedi: Metall. Mater. Trans. A, 2007, vol. 38, pp. 1417-25.

11. A. Parisi and M. Plapp: Acta Mater., 2008, vol. 56, pp. 1348-57.

12. M. Perrut, S. Akamatsu, S. Bottin-Rousseau, and G. Faivre: Phys. Rev. E, 2009, vol. 79, p. 032602.

13. M. Asta, C. Beckermann, A. Karma, W. Kurz, R. Napolitano, M. Plapp, G. Purdy, M. Rappaz, and R. Trivedi: Acta Mater., 2009, vol. 57, pp. 941-71.

14. S. Akamatsu and M. Plapp: Curr. Opin. Solid State Mater. Sci., 2016, vol. 20, pp. 46-54

15. R. Contieri, C. Rios, M. Zanotello, and R. Caram: Mater. Charact., 2008, vol. 59, pp. 693-99.

16. A. Dennstedt and L. Ratke: Trans. Indian Inst. Met., 2012, vol. 65, pp. $777-82$.
17. A. Choudhury: Trans. Indian Inst. Met., 2015, vol. 68, pp. $1137-43$.

18. J. Hötzer, M. Jainta, P. Steinmetz, B. Nestler, A. Dennstedt, A. Genau, M. Bauer, H. Köstler, and U. Rüde: Acta Mater., 2015, vol. 93, pp. 194-204.

19. J. Hötzer, P. Steinmetz, M. Jainta, S. Schulz, M. Kellner, B. Nestler, A. Genau, A. Dennstedt, M. Bauer, H. Köstler et al.: Acta Mater., 2016, vol. 106, pp. 249-59.

20. P. Steinmetz, J. Hötzer, M. Kellner, A. Dennstedt, and B. Nestler: Comput. Mater. Sci., 2016, vol. 117, pp. 205-14.

21. K.A. Jackson and J.D. Hunt: Trans. Metall. Soc. AIME, 1966, vol. 236 , pp. $1129-42$

22. M. Taylor, R. Fidler, and R. Smith: J. Cryst. Growth, 1968, vol. 3, pp. $666-73$.

23. T. Digges and R. Tauber: Metall. Trans., 1971, vol. 2, pp. 1683-89.

24. H. Kerr and M. Lewis: J. Cryst. Growth, 1972, vol. 15, pp. 117-25.

25. T. Digges, Jr. and R. Tauber: J. Cryst. Growth, 1971, vol. 8, pp. 132-34

26. M. Savas, L. Clapham, and R. Smith: J. Mater. Sci., 1990, vol. 25, pp. 909-13.

27. H. Kerr and W. Winegard: Can. Metall. Q., 1967, vol. 6, pp. 67-70.

28. M. Taylor, R. Fidler, and R. Smith: Metall. Trans., 1971, vol. 2, pp. 1793-98.

29. M. Şahin and E. Çadirli: J. Mater. Sci. Mater. Electron., 2012, vol. 23, pp. 484-92.

30. J. Bromley, F. Vnuk, and R. Smith: J. Mater. Sci., 1983, vol. 18, pp. 3143-53.

31. G. Piatti and G. Pellegrini: Journal of Materials Science, 1976, vol. 11 (5), pp. 913-924.

32. G. Beghi, G. Piatti, and K. Street: J. Mater. Sci., 1971, vol. 6, pp. $118-25$.

33. M. Notis, D. Shah, S. Young, and C. Graham: IEEE Trans. Magn., 1979, vol. 15, pp. 957-66.

34. M. Sahoo, G. Delamore, and R. Smith: J. Mater. Sci., 1980, vol. 15, pp. 1097-1103.

35. G. Nishimura, R. Fidler, M. Taylor, and R. Smith: Can. Metall. Q., 1969, vol. 8, pp. 319-22.

36. P. Taylor, H. Kerr, and W. Winegard: Can. Metall. Q., 1964 , vol. 3, pp. 235-37

37. D. Jaffrey and G. Chadwick: Metall. Trans., 1970, vol. 1, pp. 3389-96.

38. F. Vnuk, M. Sahoo, D. Baragar, and R. Smith: J. Mater. Sci., 1980, vol. 15 , pp. 2573-83.

39. H. Kaya, M. Gündüz, E. Çadirli, and O. Uzun: J. Mater. Sci., 2004, vol. 39, pp. 6571-76.

40. H. Kaya, E. Çadırlı, and M. Gündüz: J. Mater. Eng. Perform., 2003, vol. 12 , pp. 456-69.

41. Y. Goto, M. Kurosaki, and H. Esaka: J. Jpn Inst. Met., 2011, vol. 75 , pp. 392-97.

42. M. Şahin and F. Karakurt: Physica B, 2018, vol. 545, pp. 48-54.

43. K. Sharma and R. Rai: Thermochim. Acta, 2012, vol. 535, pp. 66-70.

44. B. Caroli, C. Caroli, G. Faivre, and J. Mergy: J. Cryst. Growth, 1992, vol. 118 , pp. 135-50

45. A. Valance, C. Misbah, D. Temkin, and K. Kassner: Phys. Rev. E, 1993, vol. 48, p. 1924.

46. S. Bottin-Rousseau, M. Șerefolu, S. Akamatsu, and G. Faivre: IOP Conf. Ser. Mater. Sci. Eng., 2012, vol. 27, p. 012088.

47. S. Akamatsu, S. Bottin-Rousseau, M. Şerefoğlu, and G. Faivre: Acta Mater., 2012, vol. 60, pp. 3199-3205.

48. S. Akamatsu, S. Bottin-Rousseau, M. Şerefoğlu, and G. Faivre: Acta Mater., 2012, vol. 60, pp. 3206-14.

49. S. Ghosh, A. Choudhury, M. Plapp, S. Bottin-Rousseau, G. Faivre, and S. Akamatsu: Phys. Rev. E, 2015, vol. 91, p. 022407.

50. S. Ghosh and M. Plapp: Acta Mater., 2017, vol. 140, pp. 140-48.

51. L. Rátkai, G.I. Tóth, L. Környei, T. Pusztai, and L. Gránásy: $J$. Mater. Sci., 2017, vol. 52, pp. 5544-5558.

52. A. Choudhury and B. Nestler: Phys. Rev. E, 2012, vol. 85, p. 021602.

53. M. Plapp: Phys. Rev. E, 2011, vol. 84, p. 031601.

54. R. Kobayashi: Physica D, 1993, vol. 63, pp. 410-23.

55. J.J. Eggleston, G.B. McFadden, and P.W. Voorhees: Physica D, 2001, vol. 150, pp. 91-103. 
56. P. Mathis: J. Indian Inst. Sci., 2016, vol. 96, pp. 179-98.

57. I. Ansara, A. Dinsdale, and M. Rand: Cost 507: Thermochemical Database for Light Metal Alloys, Office for Official Publications of the European Communities, Luxembourg, 1998, vol. 2.

58. A. Choudhury, M. Kellner, and B. Nestler: Curr. Opin. Solid State Mater. Sci., 2015, vol. 19, pp. 287-300.

59. J.-O. Andersson, T. Helander, L. Höglund, P. Shi B. Sundman: CALPHAD, 2002, vol. 26, pp. 273-312.

60. J. Pstruś: J. Min. Metall. B, 2017, vol. 53, pp. 309-18.

61. The HDF Group: Hierarchical Data Format, Version 5. http:// www.hdfgroup.org/HDF5/1997-NNNN.

62. J. Ahrens, B. Geveci, and C. Law: The Visualization Handbook, Academic, New York, 2005, vol. 717.

63. U. Ayachit: The Paraview Guide: A Parallel Visualization Application, Kitware, Inc., New York, 2015.

64. J.D. Hunter: Comput. Sci. Eng., 2007, vol. 9, p. 90.
65. S. Van der Walt, J.L. Schönberger, J. Nunez-Iglesias, F. Boulogne, J.D. Warner, N. Yager, E. Gouillart, and T. Yu: PeerJ, 2014, vol. 2, p. e453

66. D. Wheeler, D. Brough, T. Fast, S. Kalidindi, and A. Reid: PyMKS: materials knowledge system in Python, 2014.

67. D.B. Brough, D. Wheeler, and S.R. Kalidindi: Integr. Mater. Manuf. Innov., 2017, vol. 6, pp. 36-53.

68. A. Parisi and M. Plapp: Europhys. Lett., 2010, vol. 90, p. 26010.

69. A. Vondrous, M. Selzer, J. Hötzer, and B. Nestler: Int. J. High Perform. Comput. Appl., 2014, vol. 28, pp. 61-72.

70. B. Saatçi, N. Maraşl1, and M. Gündüz: Thermochim. Acta, 2007, vol. 454, pp. 128-134.

71. J. Hoshen and R. Kopelman: Phys. Rev. B, 1976, vol. 14, p. 3438.

Publisher's Note Springer Nature remains neutral with regard to jurisdictional claims in published maps and institutional affiliations. 